\title{
Gradient theory for plasticity via homogenization of discrete dislocations
}

Received June 25, 2008

Abstract. We deduce a macroscopic strain gradient theory for plasticity from a model of discrete dislocations.

We restrict our analysis to the case of a cylindrical symmetry for the crystal under study, so that the mathematical formulation will involve a two-dimensional variational problem.

The dislocations are introduced as point topological defects of the strain fields, for which we compute the elastic energy stored outside the so-called core region. We show that the $\Gamma$-limit of this energy (suitably rescaled), as the core radius tends to zero and the number of dislocations tends to infinity, takes the form

$$
E=\int_{\Omega}\left(W\left(\beta^{e}\right)+\varphi\left(\operatorname{Curl} \beta^{e}\right)\right) d x,
$$

where $\beta^{e}$ represents the elastic part of the macroscopic strain, and Curl $\beta^{e}$ represents the geometrically necessary dislocation density. The plastic energy density $\varphi$ is defined explicitly through an asymptotic cell formula, depending only on the elastic tensor and the class of the admissible Burgers vectors, accounting for the crystalline structure. It turns out to be positively 1-homogeneous, so that concentration on lines is permitted, accounting for the presence of pattern formations observed in crystals such as dislocation walls.

Keywords. Variational models, energy minimization, relaxation, plasticity, strain gradient theories, stress concentration, dislocations

\section{Contents}

\begin{tabular}{|c|c|}
\hline \multirow{2}{*}{\multicolumn{2}{|c|}{$\begin{array}{ll}\text { 1. } & \text { Introduction } \ldots \ldots \\
\text { 2. } & \text { Heuristic for the scaling } \\
\end{array}$}} \\
\hline & 1236 \\
\hline \begin{tabular}{l|l|l|} 
& 2.1. Self-energy & $\ldots$
\end{tabular} & 1236 \\
\hline 2.2. Long range interaction between dislocations & 1238 \\
\hline 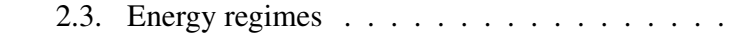 & 1239 \\
\hline 3. The setting of the problem . . & 1239 \\
\hline 4. Cell formula for the self-energy. & 1243 \\
\hline 5. A Korn type inequality for fields with prescribed curl & 12 \\
\hline
\end{tabular}

A. Garroni, M. Ponsiglione: Dipartimento di Matematica "G. Castelnuovo", Università di Roma "La Sapienza", P.le Aldo Moro 5, I-00185 Roma, Italy; e-mail: garroni@mat.uniroma1.it,ponsigli@mat.uniroma1.it

G. Leoni: Department of Mathematical Sciences, Carnegie Mellon University, Pittsburgh, PA 15213, USA; e-mail: giovanni@ andrew.cmu.edu

Mathematics Subject Classification (2010): 35R35, 49J45, 74C05, 74E10, 74E15, 74B10, 74G70 


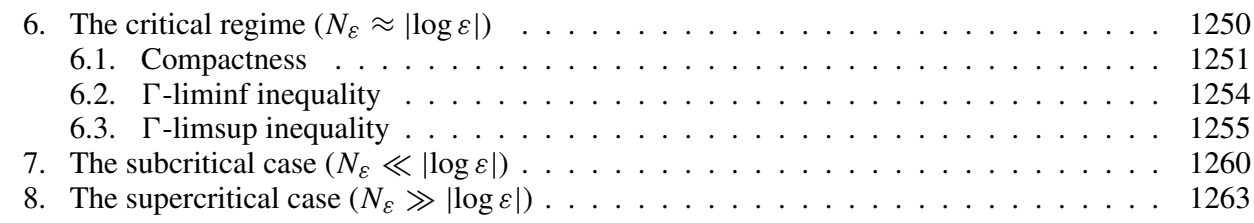

\section{Introduction}

In the last decade the study of crystal defects such as dislocations has been increasingly active. The presence of dislocations (and their motion) is indeed considered the main mechanism of plastic deformations in metals. Various phenomenological models have been proposed to account for plastic effects due to dislocations, such as the so-called strain gradient theories.

The aim of this paper is to provide a rigorous derivation of a strain gradient theory for plasticity as a mesoscopic limit of systems of discrete dislocations, which are introduced as point defects of the strain fields, for which we compute the elastic energy stored outside the so-called core region.

We focus on the stored energy, disregarding dissipation, and we restrict our analysis to the case of a cylindrical symmetry for the crystal under study, so that the mathematical formulation will involve a two-dimensional variational problem.

Many theories of plasticity are framed within linearized elasticity. In classical linear elasticity a displacement of $\Omega$ is a regular vector field $u: \Omega \rightarrow \mathbb{R}^{2}$. The equilibrium equations have the form $\operatorname{Div} \mathbb{C}[e(u)]=0$, with $\mathbb{C}$ a linear operator from $\mathbb{R}^{2 \times 2}$ into itself, and $e(u):=\frac{1}{2}\left(\nabla u+(\nabla u)^{\top}\right)$ the infinitesimal strain tensor. The corresponding elastic energy is

$$
\int_{\Omega} W(\nabla u) d x
$$

where

$$
W(\xi)=\frac{1}{2} \mathbb{C} \xi: \xi
$$

is the elastic energy density, and the elasticity tensor $\mathbb{C}$ satisfies

$$
c_{1}\left|\xi^{\mathrm{sym}}\right|^{2} \leq \mathbb{C} \xi: \xi \leq c_{2}\left|\xi^{\mathrm{sym}}\right|^{2} \quad \text { for any } \xi \in \mathbb{M}^{2 \times 2},
$$

where $\xi^{\text {sym }}:=\frac{1}{2}\left(\xi+\xi^{\top}\right)$ and $c_{1}$ and $c_{2}$ are two given positive constants. In this linear framework the presence of plastic deformations is classically modeled by the additive decomposition of the gradient of the displacement into the elastic strain $\beta^{e}$ and the plastic strain $\beta^{p}$, i.e., $\nabla u=\beta^{e}+\beta^{p}$. The elastic energy induced by a given plastic strain is then

$$
\int_{\Omega} W\left(\nabla u-\beta^{p}\right) d x .
$$

In view of their microscopic nature the presence of dislocations, responsible for the plastic deformation, can be modeled in the continuum by prescribing the curl of the field $\beta^{p}$. 
The quantity $\operatorname{Curl} \beta^{p}=\mu$ is then called Nye's dislocation density tensor (see [19]). Inspired by this idea, the strain gradient theory for plasticity, first proposed by Fleck and Hucthinson [10] and then developed by Gurtin (see for instance [13], [14]), assigns an additional phenomenological energy to the plastic deformation depending on the dislocation density, so that the stored energy looks like

$$
\int_{\Omega} W\left(\nabla u-\beta^{p}\right) d x+\int_{\Omega} \varphi\left(\operatorname{Curl} \beta^{p}\right) d x .
$$

Note that for a given preexisting strain $\beta^{p}$, the minimum of the energy in (4) (possibly subject to external loads) depends only on $\operatorname{Curl} \beta^{p}$. Hence the relevant variable in this problem is a strain field $\beta$ whose curl is prescribed. The main issue with this model is the choice of the function $\varphi$ in (5). In fact the usual choice (see for instance [15]) is to take $\varphi$ quadratic, and then to fit the free parameters through experiments as well as simulations, as in [18]. This choice has the well-known disadvantage of preventing concentration of the dislocation density, that is instead allowed, for instance, by the choice $\varphi(\operatorname{Curl} \beta):=|\operatorname{Curl} \beta|$, recently proposed by Conti and Ortiz in [7] (see also [20]).

The aim of this paper is to derive the strain gradient model (5) for the stored energy, starting from a basic model of discrete dislocations that accounts for the crystalline structure. As a consequence we will also get a formula for the function $\varphi$, determined only by the elasticity tensor $\mathbb{C}$ and the Burgers vectors of the crystal.

A purely discrete description of a crystal in the presence of dislocations can be given after introducing the discrete equivalent of $\beta^{p}$ and $\mu:=\operatorname{Curl} \beta^{p}$, following the approach of Ariza and Ortiz [1]. That model is the starting point for a microscopic description of dislocations. In the passage from discrete to continuum one can consider an intermediate (so to say) semi-discrete model known in the literature as the discrete dislocation model (see also [9], [6]), in which the atomic scale is introduced as an internal small parameter $\varepsilon$ referred to as the core radius. The gap between the purely discrete model and the discrete dislocation model can be usually filled through an interpolation procedure (see for instance [21]). In this paper, to simplify matters, we will adopt the discrete dislocation model.

In the discrete dislocation model a straight dislocation orthogonal to the cross section $\Omega$ is identified with a point $x_{0} \in \Omega$ or, more precisely, with a small region surrounding the dislocation referred to as the core region, i.e., a ball $B_{\varepsilon}\left(x_{0}\right)$, the core radius $\varepsilon$ being proportional to (the ratio between the dimensions of the crystal and) the underlying lattice spacing. The presence of the dislocation can be detected by looking at the topological singularities of a continuum strain field $\beta$, i.e.,

$$
\int_{\partial B_{\varepsilon}\left(x_{0}\right)} \beta \cdot t d s=b,
$$

where $t$ is the tangent to $\partial B_{\varepsilon}\left(x_{0}\right)$ and $b$ is the Burgers vector of the given dislocation.

We will focus our analysis on the case when there may be many dislocations crossing the domain $\Omega$. A generic distribution of $N$ dislocations will then be identified with $N$ points $\left\{x_{i}\right\}_{i=1}^{N}$ (or equivalently with the corresponding core regions), each corresponding 
to some Burgers vectors $b_{i}$ belonging to a finite set $S$ of admissible Burgers vectors, depending on the crystalline structure, e.g., for square crystals, up to a renormalization factor, $S=\left\{e_{1}, e_{2},-e_{1},-e_{2}\right\}$.

In our analysis we introduce a second small scale $\rho_{\varepsilon} \gg \varepsilon$ (the hard core radius) at which a cluster of dislocations will be identified with a multiple dislocation. In mathematical terms this corresponds to introducing the span $\mathbb{S}$ of $S$ over $\mathbb{Z}$ (i.e., the set of finite combinations of Burgers vectors with integer coefficients) and to representing a generic distribution of dislocations as a measure $\mu$ of the type

$$
\mu=\sum_{i=1}^{N} \delta_{x_{i}} \xi_{i}, \quad \xi_{i} \in \mathbb{S},
$$

where the distance between the $x_{i}$ 's is at least $2 \rho_{\varepsilon}$. The admissible strain fields $\beta$ corresponding to $\mu$ are defined outside the core region, i.e., in $\Omega \backslash \bigcup_{i} B_{\varepsilon}\left(x_{i}\right)$, and satisfy

$$
\int_{\partial B_{\varepsilon}\left(x_{i}\right)} \beta \cdot t d s=\xi_{i} \quad \text { for all } i=1, \ldots, N .
$$

It is well-known (and it will be discussed in detail below) that the plastic distortion due to the presence of dislocations decays as the inverse of the distance from the dislocations. This justifies the use of the linearized elastic energy outside the core region $\bigcup_{i} B_{\varepsilon}\left(x_{i}\right)$. On the other hand, considerations at a discrete level show that the elastic energy stored in the core region can be neglected. Therefore, the elastic energy corresponding to $\mu$ and $\beta$ is given by

$$
E_{\varepsilon}(\mu, \beta):=\int_{\Omega_{\varepsilon}(\mu)} W(\beta) d x,
$$

where $\Omega_{\varepsilon}(\mu)=\Omega \backslash \bigcup_{i} B_{\varepsilon}\left(x_{i}\right)$. By minimizing the elastic energy (7) among all strains satisfying (6), we obtain the energy induced by the dislocation $\mu$. Note that, in view of the compatibility condition (6), this residual energy is positive whenever $\mu \neq 0$.

In the case $W(\beta)=|\beta|^{2}$, with $\beta$ a curl free vector field in $\Omega \backslash \bigcup_{i} B_{\varepsilon}\left(x_{i}\right)$, this model coincides (setting $\beta=\nabla \theta, \theta$ being the phase function) with the energy proposed by Bethuel, Brezis and Hélein (see [4]) to deduce the so-called renormalized energy in the study of vortices in superconductors. In the context of dislocations this choice of $W$ and $\beta$ corresponds to a model for screw dislocations in an anti-planar setting. $\Gamma$-convergence in this framework has been studied in [21] in the energy regime $E_{\varepsilon} \approx|\log \varepsilon|$ that corresponds to a finite number of dislocations.

In the planar context of edge dislocations that we consider here, the connection with the Ginzburg-Landau model for vortices is more formal and the analysis has specific difficulties due to the vectorial nature of the circulation constraint $(6)$ and the properties of the energy density $W$. A first analysis of this model has been perfomed by Cermelli and Leoni [6] who obtain an asymptotic expansion of the energy functionals $E_{\varepsilon}$ in (7), in the case of a fixed configuration of dislocations. In particular they deduce the corresponding renormalized energy representing the effect of the Peach-Koehler force.

The purpose of this paper is to consider the energy functional $E_{\varepsilon}$ in (7), where the unknowns are the distribution of dislocations and the corresponding admissible strains, 
and to study its asymptotic behavior in terms of $\Gamma$-convergence as the lattice spacing $\varepsilon$ tends to zero and the number of dislocations $N_{\varepsilon}$ goes to infinity. Under a suitable condition on the hard core scale, we can show that in the asymptotics the energy $E_{\varepsilon}$ can be decomposed into two effects: the self-energy, concentrated in the hard core region, and the interaction energy, diffused in the remaining part of $\Omega$. The general idea is that the $\Gamma-$ limit of the (rescaled) energy functionals $E_{\varepsilon}$ as $\varepsilon$ goes to zero is given by the sum of these two effects. For different regimes for the number of dislocations $N_{\varepsilon}$ the corresponding $\Gamma$-limit will exhibit the dominance of one of the two effects: the self-energy is predominant for $N_{\varepsilon} \ll|\log \varepsilon|$, while the interaction energy is predominant for $N_{\varepsilon} \gg|\log \varepsilon|$. In a critical regime $N_{\varepsilon} \approx|\log \varepsilon|$ the two effects will be balanced and the structure of the limiting energy is

$$
\int_{\Omega} W(\beta) d x+\int_{\Omega} \varphi\left(\frac{d \mu}{d|\mu|}\right) d|\mu|
$$

The first term is the elastic energy of the limiting rescaled strain and comes from the interaction energy. The second term represents the plastic energy and comes from the self-energy; it depends only on the rescaled dislocation density $\mu=\operatorname{Curl} \beta$ through a positively 1-homogeneous density function $\varphi$, defined by a suitable asymptotic cell problem formula. The constraint Curl $\beta=\mu$ comes from the admissibility condition (6) that the admissible strains have to satisfy at a discrete level.

If $(\beta, \mu)$ is a configuration that makes the continuous energy in $(8)$ finite, then necessarily $\beta^{\text {sym }}$ belongs to $L^{2}\left(\Omega ; \mathbb{M}^{2 \times 2}\right)$, and $\mu$ has finite mass. A natural question arises: does $\mu$ belong to the space $H^{-1}\left(\Omega ; \mathbb{R}^{2}\right)$ ? We give a positive answer to this problem proving a Korn type inequality for fields whose curl is a prescribed measure, based on a fine estimate for elliptic systems with $L^{1}$ data recently proved by Bourgain, Brezis and Van Schaftingen (see [3], [5]). In particular we deduce that concentration of dislocations on lines is permitted, accounting for the presence of pattern formations observed in crystals such as dislocation walls, while concentration on points is not permitted. An additional feature of the limit energy is the anisotropy of the self-energy density inherited from the anisotropic elastic tensor and the class of the admissible Burgers vectors accounting for the crystalline structure.

The idea of deducing continuous models by homogenization of lower dimensional singularities has been used in other contexts. Our result is very much in the spirit of earlier results on the asymptotic analysis for the Ginzburg-Landau model for superconductivity as the number of vortices goes to infinity, done by Jerrard and Soner in [16], and by Sandier and Serfaty in [23] (see also [24] and the references therein).

As for the study of dislocations, a similar analysis was done by Garroni and Focardi [11] starting from a phase field model introduced by Koslowski, Cuitiño and Ortiz [17] and inspired by the Peierls-Nabarro model (see also [12]).

The plan of the paper is the following. In Section 2 we compute heuristically the asymptotic behavior of the self-energy and the interaction energy in terms of the number of dislocations $N_{\varepsilon}$ and the core radius $\varepsilon$. In Section 3 we introduce rigorously the mathematical setting of the problem, defining the class of admissible configurations of dislocations, the class of the corresponding admissible strains and the rescaled energy functionals. In Section 4 we provide the asymptotic cell problem formulas defining the 
density $\varphi$ of the plastic energy. In Section 5 we prove a Korn type inequality for fields with prescribed curl. In Section 6 we give our main result concerning the $\Gamma$-convergence of the energy functionals in the critical regime $N_{\varepsilon} \approx|\log \varepsilon|$, while Sections 7 and 8 are devoted to the subcritical case and the supercritical case respectively.

\section{Heuristic for the scaling}

In this section we identify the energy regimes, as the internal scale $\varepsilon$ tends to zero, of the elastic energy induced by a distribution of dislocations $\mu_{\varepsilon}:=\sum_{i=1}^{N_{\varepsilon}} \xi_{i} \delta_{x_{i}}$, where we omit the dependence on $\varepsilon$ of the $x_{i}$ 's. The idea is that if the scaling of the energy of a given sequence of distributions of dislocations is assigned, this provides a bound for $N_{\varepsilon}$. In this section, in order to identify the relevant energy regimes, we do the converse: we assume the number of dislocations $N_{\varepsilon}$ present in the crystal is known, and we compute (heuristically) the corresponding energy behavior as $\varepsilon$ goes to zero.

As already mentioned in the introduction, the general idea is that the energy will always be given by the sum of two terms: the self-energy, concentrated in a small region (the hard core region) surrounding the dislocations, and the interaction energy, diffused in the remaining part of the domain $\Omega$. We will first introduce and compute heuristically the asymptotic behavior of these two terms separately. Then we will identify the predominant term (which depends on the behavior of $N_{\varepsilon}$ ) between these two energies.

\subsection{Self-energy}

The self-energy of a distribution of dislocations $\mu_{\varepsilon}:=\sum_{i=1}^{N_{\varepsilon}} \xi_{i} \delta_{x_{i}}$ is essentially given by the sum of the energies that would be induced by a single dislocation $\mu_{\varepsilon}^{i}:=\xi_{i} \delta_{x_{i}}$, $i=1, \ldots, N_{\varepsilon}$ (i.e., if no other dislocations $\xi_{j} \delta_{x_{j}}, j \neq i$, were present in the crystal). As we will see, the self-energy of a single dislocation $b \delta_{x}$ is asymptotically (as $\varepsilon \rightarrow 0$ ) independent of the position of the dislocation point $x$ in $\Omega$, depending only on the elasticity tensor $\mathbb{C}$ and on the Burgers vector $b$.

We begin by computing the self-energy in a very simple situation. We assume for the time being that $\Omega$ is the ball $B_{1}$ of radius one and center zero, that $\mu_{\varepsilon} \equiv \mu=b \delta_{0}$ with $|b|=1$, and we compute the energy of an admissible strain $\beta$ considering the toy energy given by the square of the $L^{2}$ norm of $\beta$. More precisely, we consider

$$
E_{\varepsilon}^{\mathrm{toy}}(\mu, \beta):=\int_{B_{1} \backslash B_{\varepsilon}}|\beta|^{2} d x
$$

and the self-energy induced by $\mu$ given by minimizing this energy among all admissible strains $\beta$ compatible with $\mu_{\varepsilon}$, i.e., satisfying

$$
\int_{\partial B_{\varepsilon}} \beta \cdot t d s=b .
$$


In this setting the minimum strain $\beta(\mu)$ can be computed explicitly, and in polar coordinates it is given by the expression

$$
\beta(\mu)(r, \theta):=\frac{1}{2 \pi r} t(r, \theta) \otimes b,
$$

where $t(r, \theta)$ is the tangent unit vector to $\partial B_{r}(0)$ at the point $(r, \theta)$. We deduce the following expression for the self-energy stored in $B_{1} \backslash B_{\varepsilon}$ :

$$
E_{\varepsilon}^{\text {self }}:=\int_{\varepsilon}^{1} \frac{1}{2 \pi r} d r=\frac{1}{2 \pi}(\log 1-\log \varepsilon) .
$$

Hence, as $\varepsilon \rightarrow 0$ the self-energy of a single dislocation behaves like $|\log \varepsilon|$.

Notice that most of the self-energy is concentrated around a small region surrounding the dislocation. Indeed, fix $0<s<1$, and compute the energy stored in the ball $B_{\varepsilon^{s}}(0)$,

$$
\begin{aligned}
\int_{B_{\varepsilon^{s}}(0)}|\beta(\mu)|^{2} d x & =\int_{\varepsilon}^{\varepsilon^{s}} \frac{1}{2 \pi r} d r=\frac{1}{2 \pi}\left(\log \left(\varepsilon^{s}\right)-\log \varepsilon\right)=\frac{1}{2 \pi}(1-s)|\log \varepsilon| \\
& =(1-s) E_{\varepsilon}^{\text {self }} .
\end{aligned}
$$

As we will see, in view of the Korn inequality, the logarithmic behavior and the concentration phenomenon of the self-energy hold true also in the case of elastic energies depending only on $\beta^{\text {sym }}$ as in (7). In this respect the position of the dislocation and the shape of $\Omega$ itself do not have a big impact on the value of the self-energy. It then seems convenient to introduce the self-energy as a quantity depending only on the Burgers vector $b$ (and the elasticity tensor $\mathbb{C}$ ) through a cell problem. Before doing that, we proceed heuristically, by introducing the notion of hard core of the dislocation $\delta_{0}$, as a region surrounding the dislocation such that

(i) the hard core region contains almost all the self-energy;

(ii) the hard core region shrinks at the dislocation point as the atomic scale $\varepsilon$ tends to zero.

To this end, in view of the previous discussion, it is enough to define the core region as $B_{\rho_{\varepsilon}}(0)$, where the hard core radius $\rho_{\varepsilon}$ satisfies

(i) $\lim _{\varepsilon \rightarrow 0} \rho_{\varepsilon} / \varepsilon^{s}=\infty$ for every fixed $0<s<1$;

(ii) $\rho_{\varepsilon}^{2} \rightarrow 0$ as $\varepsilon \rightarrow 0$.

Note that condition (i) is indeed equivalent to

(i') $\lim _{\varepsilon \rightarrow 0} \log \rho_{\varepsilon} / \log \varepsilon=0$.

A direct consequence of our definitions is that the self-energy can be identified (up to lower order terms) with the elastic energy stored in the hard core region, for which, with a little abuse, we will use the same notation, i.e.,

$$
E_{\varepsilon}^{\text {self }}:=\int_{B_{\rho_{\varepsilon}}(0)}|\beta(\mu)|^{2} d x .
$$


Consider next the case of a generic configuration of dislocations $\mu_{\varepsilon}:=\sum_{i=1}^{N_{\varepsilon}} b \delta_{x_{i}}$ in $\Omega$ (but for simplicity we will keep the Burgers vector equal to $b$ and the toy elastic energy (9)). The hard core region of $\mu_{\varepsilon}$ is given by the union of the balls $B_{\rho_{\varepsilon}}\left(x_{i}\right)$.

Again we require a decay for $\rho_{\varepsilon}$ and that the area of the hard core region tends to zero as $\varepsilon \rightarrow 0, N_{\varepsilon} \rightarrow \infty$, i.e.,

(i) $\lim _{\varepsilon \rightarrow 0} \rho_{\varepsilon} / \varepsilon^{s}=\infty$ for every fixed $0<s<1$;

(ii) $N_{\varepsilon} \rho_{\varepsilon}^{2} \rightarrow 0$ as $\varepsilon \rightarrow 0$.

Moreover, we require that the dislocations are separated by a distance $2 \rho_{\varepsilon}$, i.e.,

(iii) the balls $B_{\rho_{\varepsilon}}\left(x_{i}\right)$ are pairwise disjoint.

This condition motivates the name of the hard core region. In view of this assumption, it is natural to identify the self-energy of $\mu_{\varepsilon}$ as the elastic energy stored in the hard core region. Therefore, we set

$$
E_{\varepsilon}^{\text {self }}\left(\mu_{\varepsilon}\right):=\int_{\text {Hard Core }}\left|\beta\left(\mu_{\varepsilon}\right)\right|^{2} d x=\sum_{i=1}^{N_{\varepsilon}} \int_{B_{\rho_{\varepsilon}}\left(x_{i}\right)}\left|\beta\left(\mu_{\varepsilon}\right)\right|^{2} d x
$$

We expect each term of the sum on the right hand side to be asymptotically equivalent to the self-energy of a single dislocation introduced in (11), so that in view of (10),

$$
E_{\varepsilon}^{\text {self }}\left(\mu_{\varepsilon}\right)=\sum_{i=1}^{N_{\varepsilon}} \int_{B_{\rho_{\varepsilon}}\left(x_{i}\right)}\left|\beta\left(\mu_{\varepsilon}\right)\right|^{2} d x \approx N_{\varepsilon}|\log \varepsilon| .
$$

This expression represents the asymptotic behavior of the self-energy as $\varepsilon \rightarrow 0, N_{\varepsilon} \rightarrow \infty$.

\subsection{Long range interaction between dislocations}

Since the self-energy is concentrated in a small region surrounding the dislocations, it is natural to define the interaction energy $E_{\varepsilon}^{\text {inter }}$ as the energy diffused in the remaining part of the domain, far from each dislocation. In view of this definition the total energy $E_{\varepsilon}\left(\mu_{\varepsilon}, \beta\left(\mu_{\varepsilon}\right)\right)$ is given by the sum of the self-energy and the interaction energy:

$$
\begin{aligned}
E_{\varepsilon}\left(\mu_{\varepsilon}, \beta\left(\mu_{\varepsilon}\right)\right)= & \int_{\Omega_{\varepsilon}\left(\mu_{\varepsilon}\right)} W\left(\beta\left(\mu_{\varepsilon}\right)\right) d x \\
& =\int_{\text {Hard Core }} W\left(\beta\left(\mu_{\varepsilon}\right) d x+\int_{\Omega \backslash \text { Hard Core }} W\left(\beta\left(\mu_{\varepsilon}\right)\right) d x\right. \\
& =E_{\varepsilon}^{\text {self }}\left(\mu_{\varepsilon}\right)+E_{\varepsilon}^{\text {inter }}\left(\mu_{\varepsilon}\right),
\end{aligned}
$$

where we recall that $\Omega_{\varepsilon}\left(\mu_{\varepsilon}\right)$ is the region of $\Omega$ outside the dislocation cores, that is, $\Omega_{\varepsilon}\left(\mu_{\varepsilon}\right):=\Omega \backslash \bigcup_{i=1}^{N_{\varepsilon}} B_{\varepsilon}\left(x_{i}\right)$, for $\mu_{\varepsilon}=\sum_{i=1}^{N_{\varepsilon}} \delta_{x_{i}} b$. Let us compute heuristically the interaction energy for a very simple configuration of dislocations and for the toy energy considered in 9 . Let $\Omega$ be the unit ball $B_{1}$, and $\mu_{\varepsilon}$ be a configuration of $N_{\varepsilon}$ periodically 
distributed dislocations whose Burgers vector $b$ has modulus one. The stored interaction energy is given by

$$
E_{\varepsilon}^{\text {inter }}\left(\mu_{\varepsilon}, \beta\left(\mu_{\varepsilon}\right)\right):=\int_{\Omega \backslash \text { Hard Core }}\left|\beta\left(\mu_{\varepsilon}\right)\right|^{2} d x=\int_{0}^{1} d r \int_{0}^{2 \pi r} \chi_{\varepsilon}|\beta(r, \theta)|^{2} d \theta,
$$

where $\chi_{\varepsilon}$ denotes the characteristic function of the set $\Omega \backslash$ Hard Core. Thanks to the uniform distribution of the $x_{i}$ 's in $B_{1}=\Omega$ we deduce that the number of dislocations contained in each ball of radius $r$ is proportional to the area of the ball, and more precisely is of order $\pi r^{2} N_{\varepsilon}$. Therefore, the average $\bar{\beta}_{t}(r)$ of the tangential component of the strain on each circle $\partial B_{r}$ outside the dislocation hard cores, i.e., of $\beta_{t}(r, \theta) \chi_{\varepsilon}$, is of order $\pi r^{2} N_{\varepsilon} / 2 \pi r=N_{\varepsilon} r / 2$. The error is small thanks to the fact that the area of the hard cores region is negligible. By Jensen's inequality we obtain the following estimate from below for the interaction energy:

$$
\begin{aligned}
E_{\varepsilon}^{\text {inter }}\left(\mu_{\varepsilon}\right) & =\int_{0}^{1} \int_{0}^{2 \pi r} \chi_{\varepsilon}|\beta(r, \theta)|^{2} d \theta d r \geq \int_{0}^{1} 2 \pi r\left|\bar{\beta}_{t}(r)\right|^{2} d r \approx \int_{0}^{1} 2 \pi\left(N_{\varepsilon}^{2} r^{3} / 4\right) d r \\
& =C N_{\varepsilon}^{2},
\end{aligned}
$$

where $C$ is a constant independent of $\varepsilon$.

Note that the self-energy has been estimated by looking at the circulation condition that the strain $\beta_{\varepsilon}(\mu)$ has to satisfy (in order to be an admissible strain) on the circles $\partial B_{r}\left(x_{i}\right)$ for $\varepsilon \leq r \leq \rho_{\varepsilon}$, while the estimate for the interaction energy has been established by looking at the circulation condition on all circles $\partial B_{r}$ for $r \leq 1$. We will see that this estimate is indeed sharp for recovery sequences in $\Gamma$-convergence. We will then conclude that the interaction energy behaves like $N_{\varepsilon}^{2}$ as $\varepsilon \rightarrow 0, N_{\varepsilon} \rightarrow \infty$.

\subsection{Energy regimes}

In view of the heuristic arguments of the previous section we fix a function $\varepsilon \mapsto N_{\varepsilon}$ that represents the number of dislocations present in the crystal corresponding to the internal scale $\varepsilon$. The above considerations can be summarized in three regimes for the behavior of $N_{\varepsilon}$ with respect to $\varepsilon \rightarrow 0$ :

1. Dilute dislocations $\left(N_{\varepsilon} \ll|\log \varepsilon|\right)$ : in this regime the self-energy, which is of order $N_{\varepsilon}|\log \varepsilon|$, is predominant with respect to the interaction energy.

2. Critical regime $\left(N_{\varepsilon} \approx|\log \varepsilon|\right)$ : in this regime the self-energy and the interaction energy are both of order $N_{\varepsilon}|\log \varepsilon| \approx|\log \varepsilon|^{2}$.

3. Supercritical regime $\left(N_{\varepsilon} \gg|\log \varepsilon|\right)$ : in this regime the interaction energy, which is of order $\left|N_{\varepsilon}\right|^{2}$, is predominant with respect to the self-energy.

\section{The setting of the problem}

In this section we specify the mathematical setting of the problem. In particular, we introduce the class $X_{\varepsilon}$ of admissible configurations of dislocations, the class $\mathcal{A S}_{\varepsilon}(\mu)$ of the corresponding admissible strains, and the rescaled energy functionals $\mathcal{F}_{\varepsilon}$. 
From now on $\Omega$ is a bounded open subset of $\mathbb{R}^{2}$ with Lipschitz continuous boundary, representing a horizontal section of an infinite cylindrical crystal. For the given crystal, we introduce the class of Burgers vectors $S:=\left\{b_{1}, \ldots, b_{s}\right\}$. In what follows we assume that $S$ contains at least two (independent) vectors, so that

$$
\operatorname{Span}_{\mathbb{R}} S=\mathbb{R}^{2}
$$

and this will imply that the function $\varphi$ in the energy 8 is finite in the whole of $\mathbb{R}^{2}$. The case of only one Burgers vector is easier and it implies that $\varphi$ is finite only on a one-dimensional subspace of $\mathbb{R}^{2}$. We denote by $\mathbb{S}$ the span of $S$ with integer coefficients $\left(\mathbb{S}:=\operatorname{Span}_{\mathbb{Z}} S\right)$, i.e., the set of Burgers vectors for "multiple dislocations".

As in Section 2, $N_{\varepsilon}$ represents the number of dislocations present in the crystal, corresponding to the internal scale $\varepsilon$. We also introduce the sequence $\rho_{\varepsilon}$ representing the radius of the hard core surrounding the dislocations, and we require

(i) $\lim _{\varepsilon \rightarrow 0} \rho_{\varepsilon} / \varepsilon^{s}=\infty$ for every fixed $0<s<1$;

(ii) $\lim _{\varepsilon \rightarrow 0}\left|N_{\varepsilon}\right| \rho_{\varepsilon}^{2}=0$.

Condition (i) says that the hard core region contains almost all the self-energy, while condition (ii) says that the area of the hard core region tends to zero, and hence that its complement contains almost all the interaction energy. Note that conditions (i) and (ii) are compatible whenever

$$
N_{\varepsilon} \varepsilon^{s} \rightarrow 0 \quad \text { for every fixed } s>0
$$

We assume that the distance between any pair of dislocation points is at least $2 \rho_{\varepsilon}$ and we define the class $X_{\varepsilon}$ of admissible dislocations by

$$
\begin{array}{r}
X_{\varepsilon}:=\left\{\mu \in \mathcal{M}\left(\Omega ; \mathbb{R}^{2}\right): \mu=\sum_{i=1}^{M} \xi_{i} \delta_{x_{i}}, M \in \mathbb{N}, B_{\rho_{\varepsilon}}\left(x_{i}\right) \subset \Omega,\right. \\
\left.\left|x_{j}-x_{k}\right| \geq 2 \rho_{\varepsilon} \text { for every } j \neq k, \xi_{i} \in \mathbb{S}\right\},
\end{array}
$$

where $\mathcal{M}\left(\Omega ; \mathbb{R}^{2}\right)$ denotes the space of vector valued Radon measures. Given $\mu \in X_{\varepsilon}$ and $r>0$, we define

$$
\Omega_{r}(\mu):=\Omega \backslash \bigcup_{x_{i} \in \operatorname{supp}(\mu)} \bar{B}_{r}\left(x_{i}\right),
$$

where $B_{r}\left(x_{i}\right)$ denotes the open ball of center $x_{i}$ and radius $r$.

The class of admissible strains associated with any $\mu:=\sum_{i=1}^{M} \xi_{i} \delta_{x_{i}} \in X_{\varepsilon}$ is then

$$
\begin{aligned}
\left\{\beta \in L^{2}\left(\Omega_{\varepsilon}(\mu) ; \mathbb{M}^{2 \times 2}\right): \operatorname{Curl} \beta=\right. & 0 \text { in } \Omega_{\varepsilon}(\mu) \text { and } \\
& \left.\int_{\partial B_{\varepsilon}\left(x_{i}\right)} \beta \cdot t d s=\xi_{i} \text { for all } i=1, \ldots, M\right\} .
\end{aligned}
$$


Here $t$ denotes the tangent to $\partial B_{\varepsilon}\left(x_{i}\right)$ and the integrand $\beta \cdot t$ is understood in the sense of traces (see Theorem 2, page 204 in [8] and Remark 1 for more details). In the arguments below it will be useful to extend the admissible strains to the whole of $\Omega$. There are various extensions that can be considered and that are compatible with the model that we have in mind. We decide to extend the $\beta$ 's by setting their value to be zero in the dislocation cores. Thus, from now on the class $\mathcal{A S}_{\varepsilon}(\mu)$ of admissible strains associated with any $\mu:=\sum_{i=1}^{M} \xi_{i} \delta_{x_{i}}$ is given by

$$
\begin{aligned}
& \mathcal{A S}_{\varepsilon}(\mu):=\left\{\beta \in L^{2}\left(\Omega ; \mathbb{M}^{2 \times 2}\right): \beta \equiv 0 \text { in } \Omega \backslash \Omega_{\varepsilon}(\mu), \text { Curl } \beta=0 \text { in } \Omega_{\varepsilon}(\mu),\right. \\
& \left.\int_{\partial B_{\varepsilon}\left(x_{i}\right)} \beta \cdot t d s=\xi_{i} \text {, and } \int_{\Omega_{\varepsilon}(\mu)}\left(\beta-\beta^{T}\right) d x=0 \text { for all } i=1, \ldots, M\right\} .
\end{aligned}
$$

In view of the definition of the elastic energy, the last condition in the definition of $\mathcal{A} \mathcal{S}_{\varepsilon}(\mu)$ is not restrictive and it is there to guarantee uniqueness of the minimizing strain.

Remark 1. Let $\beta \in \mathcal{A S}_{\varepsilon}(\mu)$. Formally $\operatorname{Curl} \beta$ is a tensor defined by

$$
(\operatorname{Curl} \beta)_{i j l}:=\frac{\partial}{\partial x_{l}} \beta_{i j}-\frac{\partial}{\partial x_{j}} \beta_{i l},
$$

which by definition is antisymmetric with respect to the entries $j, l$. Therefore $\operatorname{curl} \beta_{(i)}$ (where $\beta_{(i)}$ denotes the $i^{\text {th }}$ row of $\beta$ ) can be identified with the scalar

$$
\operatorname{curl} \beta_{(i)}:=\frac{\partial}{\partial x_{1}} \beta_{i 2}-\frac{\partial}{\partial x_{2}} \beta_{i 1} .
$$

In the sense of distributions, $\operatorname{curl} \beta_{(i)}$ is given by

$$
\left\langle\operatorname{curl} \beta_{(i)}, \varphi\right\rangle=\int_{\Omega}\left\langle\beta_{(i)}, J \nabla \varphi\right\rangle,
$$

where $J$ is the clockwise rotation of $90^{\circ}$. From $(18)$ it turns out that whenever $\beta_{(i)}$ is in $L^{2}\left(\Omega ; \mathbb{R}^{2}\right)$, then curl $\beta_{(i)}$ is well defined for $\varphi \in H^{1}(\Omega)$ and acts continuously on it; so that

$$
\operatorname{curl} \beta_{(i)} \in H^{-1}(\Omega) \quad \text { for every } \beta \in \mathcal{A S}_{\varepsilon}(\mu) .
$$

On the other hand, if $\beta_{(i)} \in L^{1}\left(\Omega ; \mathbb{R}^{2}\right)$ and $\operatorname{curl} \beta_{(i)} \in H^{-1}(\Omega)$ then $\beta_{(i)}$ is in $L^{2}\left(\Omega ; \mathbb{R}^{2}\right)$ modulo gradients.

Finally, notice that for every $\mu:=\sum_{i=1}^{M} \xi_{i} \delta_{x_{i}} \in X_{\varepsilon}$, the circulation condition in (17) can be stated in the following equivalent way:

$$
\langle\operatorname{Curl} \beta, \varphi\rangle=\sum_{i=1}^{M} \xi_{i} c_{i}
$$

for every $\varphi \in H_{0}^{1}(\Omega)$ such that $\varphi \equiv c_{i}$ in $B_{\varepsilon}\left(x_{i}\right)$. In particular, if $\varphi$ belongs also to $C_{0}^{0}(\Omega)$, we have

$$
\langle\operatorname{Curl} \beta, \varphi\rangle=\int_{\Omega} \varphi d \mu .
$$


The elastic energy $E_{\varepsilon}$ corresponding to a pair $(\mu, \beta)$, with $\mu \in X_{\varepsilon}$ and $\beta \in \mathcal{A} \mathcal{S}_{\varepsilon}(\mu)$, is defined by

$$
E_{\varepsilon}(\mu, \beta):=\int_{\Omega_{\varepsilon}(\mu)} W(\beta) d x
$$

where

$$
W(\xi):=\frac{1}{2} \mathbb{C} \xi: \xi
$$

is the strain energy density, and the elasticity tensor $\mathbb{C}$ satisfies

$$
c_{1}\left|\xi^{\text {sym }}\right|^{2} \leq \mathbb{C} \xi: \xi \leq c_{2}\left|\xi^{\text {sym }}\right|^{2} \quad \text { for any } \xi \in \mathbb{M}^{2 \times 2},
$$

where $\xi^{\mathrm{sym}}:=\frac{1}{2}\left(\xi+\xi^{\top}\right)$ and $c_{1}$ and $c_{2}$ are two given positive constants. Since $\beta$ is always extended by zero outside $\Omega_{\varepsilon}(\mu)$, we can rewrite the energy as

$$
E_{\varepsilon}(\mu, \beta)=\int_{\Omega} W(\beta) d x .
$$

Note that for any given $\mu \in X_{\varepsilon}$, the problem

$$
\min _{\beta \in \mathcal{A S}_{\varepsilon}(\mu)} \int_{\Omega_{\varepsilon}(\mu)} W(\beta) d x
$$

has a unique solution. The proof of this fact can be obtained by removing a finite number of segments from $\Omega_{\varepsilon}(\mu)$, obtaining in such a way a simply connected domain where the Korn inequality is applied to curl-free admissible strains, and then following the direct method of calculus of variations.

We conclude by giving the notation of the rescaled functionals in the different regimes for $N_{\varepsilon}$, according to the asymptotic analysis given in the previous section:

- Subcritical or dilute regime: $N_{\varepsilon} \ll|\log \varepsilon|$. In this case the predominant contribution comes from the self-energy and is of order $N_{\varepsilon}|\log \varepsilon|$. Therefore, we set

$$
\mathcal{F}_{\varepsilon}^{\text {dilute }}(\mu, \beta):= \begin{cases}\frac{1}{N_{\varepsilon}|\log \varepsilon|} E_{\varepsilon}(\mu, \beta) & \text { if } \mu \in X_{\varepsilon}, \beta \in \mathcal{A S}_{\varepsilon}(\mu), \\ \infty & \text { otherwise in } L^{2}\left(\Omega ; \mathbb{M}^{2 \times 2}\right) .\end{cases}
$$

- Critical regime: $N_{\varepsilon}=|\log \varepsilon|$. In this case both energies are of order $N_{\varepsilon}|\log \varepsilon|=$ $|\log \varepsilon|^{2}$. Therefore, we set

$$
\mathcal{F}_{\varepsilon}(\mu, \beta):= \begin{cases}\frac{1}{|\log \varepsilon|^{2}} E_{\varepsilon}(\mu, \beta) & \text { if } \mu \in X_{\varepsilon}, \beta \in \mathcal{A S}_{\varepsilon}(\mu), \\ \infty & \text { otherwise in } L^{2}\left(\Omega ; \mathbb{M}^{2 \times 2}\right)\end{cases}
$$

- Supercritical regime: $N_{\varepsilon} \gg|\log \varepsilon|$. In this case the interaction energy is the predominant term, and is of order $N_{\varepsilon}^{2}$. Therefore, we set

$$
\mathcal{F}_{\varepsilon}^{\text {super }}(\mu, \beta):= \begin{cases}\frac{1}{N_{\varepsilon}^{2}} E_{\varepsilon}(\mu, \beta) & \text { if } \mu \in X_{\varepsilon}, \beta \in \mathcal{A} \mathcal{S}_{\varepsilon}(\mu), \\ \infty & \text { otherwise in } L^{2}\left(\Omega ; \mathbb{M}^{2 \times 2}\right) .\end{cases}
$$


Remark 2. The choice of representing the curl constraint by defining the measure $\mu$ as a sum of Dirac masses concentrated in the dislocation points is one of the possible choices. Other possibilities would be to consider more regular measures with the same mass, such as

$$
\tilde{\mu}=\sum_{i=1}^{M} \frac{\chi_{B_{\varepsilon}\left(x_{i}\right)}}{\pi \varepsilon^{2}} \xi\left(x_{i}\right)
$$

or

$$
\hat{\mu}=\sum_{i=1}^{M} \frac{\mathcal{H}^{1}\left\llcorner\partial B_{\varepsilon}\left(x_{i}\right)\right.}{2 \pi \varepsilon} \xi\left(x_{i}\right)
$$

The advantage of these alternative choices is that $\tilde{\mu}$ and $\hat{\mu}$ belong to $H^{-1}\left(\Omega ; \mathbb{R}^{2}\right)$, which is the natural space for $\operatorname{Curl} \beta$. Indeed, since $\tilde{\mu}$ ( $\hat{\mu}$ respectively) is in $H^{-1}\left(\Omega ; \mathbb{R}^{2}\right)$, we could rewrite the class of admissible strains as follows:

$$
\left\{\beta \in L^{1}\left(\Omega ; \mathbb{M}^{2 \times 2}\right): \operatorname{Curl} \beta=\tilde{\mu} \text { (respectively } \hat{\mu} \text { ) in } \Omega\right\} .
$$

This notion of admissible strains does not coincide with that given in (17), but it turns out to be equivalent to (17), in terms of $\Gamma$-convergence, in the study of the asymptotic behavior of the energy functionals as $\varepsilon \rightarrow 0$.

\section{Cell formula for the self-energy}

The self-energy stored in a neighborhood of a dislocation is of order $|\log \varepsilon|$ and, in view of the concentration of the energy, it is asymptotically not affected by the shape of the domain, depending only on the elasticity tensor $\mathbb{C}$ and on the Burgers vector $b$. It then seems natural to introduce rigorously the notion of self-energy through a cell problem.

In the following we will consider the self-energy of any multiple Burgers vector $\xi \in \mathbb{S}$. For convenience we will introduce all the quantities we need for a generic vector $\xi \in \mathbb{R}^{2}$.

For every $\xi \in \mathbb{R}^{2}$ and for all $0<r_{1}<r_{2} \in \mathbb{R}$, let

$$
\mathcal{A S}_{r_{1}, r_{2}}(\xi):=\left\{\beta \in L^{2}\left(B_{r_{2}} \backslash B_{r_{1}} ; \mathbb{M}^{2 \times 2}\right): \operatorname{Curl} \beta=0, \int_{\partial B_{r_{1}}} \beta \cdot t d s=\xi\right\},
$$

where $B_{r}$ denotes the ball of radius $r$ and center 0 .

We first note that the admissibility conditions above on a strain $\beta$ ensure an a priori bound from below for its energy. This is made precise by the following remark.

Remark 3. Given $0<r_{1}<r_{2}$ and $\xi \in \mathbb{R}^{2}$, for every admissible configuration $\beta \in$ $\mathcal{A S}_{r_{1}, r_{2}}(\xi)$ we have

$$
\int_{B_{r_{2}} \backslash B_{r_{1}}}\left|\beta^{\mathrm{sym}}\right|^{2} d x \geq c|\xi|^{2},
$$

where the constant $c$ depends only on $r_{1}$ and $r_{2}$.

Indeed, by introducing a cut with a segment $L$, the set $\left(B_{r_{2}} \backslash B_{r_{1}}\right) \backslash L$ becomes simply connected. Since $\operatorname{Curl} \beta=0$ in $\left(B_{r_{2}} \backslash B_{r_{1}}\right) \backslash L$, there exists a function $u \in$ 
$H^{1}\left(\left(B_{r_{2}} \backslash B_{r_{1}}\right) \backslash L ; \mathbb{R}^{2}\right)$ such that $\nabla u=\beta$ in $\left(B_{r_{2}} \backslash B_{r_{1}}\right) \backslash L$. By the classical Korn inequality applied to $u$ we obtain

$$
\int_{B_{r_{2}} \backslash B_{r_{1}}}|\beta-A|^{2} d x \leq C \int_{B_{r_{2}} \backslash B_{r_{1}}}\left|\beta^{\text {sym }}\right|^{2} d x
$$

for some skew symmetric matrix $A$. Moreover, since $\beta \in \mathcal{A S}_{r_{1}, r_{2}}\left(\eta_{n}\right)$, we conclude

$$
\begin{aligned}
\int_{B_{r_{2}} \backslash B_{r_{1}}}|\beta-A|^{2} d x & \geq \int_{r_{1}}^{r_{2}} \frac{1}{2 \pi \rho}\left|\int_{\partial B_{\rho}}(\beta-A) \cdot t d s\right|^{2} d \rho \\
& =\int_{r_{1}}^{r_{2}} \frac{1}{2 \pi \rho}\left|\int_{\partial B_{\rho}} \beta \cdot t d s\right|^{2} d \rho=\int_{r_{1}}^{r_{2}} \frac{1}{2 \pi \rho}|\xi|^{2} d \rho=\frac{|\xi|^{2} \log \frac{r_{2}}{r_{1}}}{2 \pi} .
\end{aligned}
$$

Set now $C_{\varepsilon}:=B_{1} \backslash B_{\varepsilon}$, and let $\psi_{\varepsilon}: \mathbb{R}^{2} \rightarrow \mathbb{R}$ be the function defined through the following cell problem:

$$
\psi_{\varepsilon}(\xi):=\frac{1}{|\log \varepsilon|} \min _{\beta \in \mathcal{A S}_{\varepsilon, 1}(\xi)} \int_{C_{\varepsilon}} W(\beta) d x \quad \text { for every } \xi \in \mathbb{R}^{2} .
$$

By (20), it is easy to see that problem (27) has a solution. We will denote by $\beta_{\varepsilon}(\xi)$ the (unique) solution of the cell problem (27) whose average is a symmetric matrix. Note that $\beta_{\varepsilon}(\xi)$ satisfies the boundary value problem

$$
\begin{cases}\operatorname{Div} \mathbb{C} \beta_{\varepsilon}(\xi)=0 & \text { in } C_{\varepsilon} \\ \mathbb{C} \beta_{\varepsilon}(\xi) \cdot v=0 & \text { on } \partial C_{\varepsilon} .\end{cases}
$$

Moreover, we will denote by $\beta_{\mathbb{R}^{2}}(\xi): \mathbb{R}^{2} \rightarrow \mathbb{R}^{2}$ the planar strain defined in all $\mathbb{R}^{2}$ corresponding to the dislocation centered at 0 with Burgers vector $\xi$. The strain $\beta_{\mathbb{R}^{2}}(\xi)$ is of the type

$$
\beta_{\mathbb{R}^{2}}(\xi)(r, \theta)=\frac{1}{r} \Gamma_{\xi}(\theta),
$$

where the function $\Gamma$ depends on the elastic properties of the crystal, namely on the elasticity tensor $\mathbb{C}$, and $\beta_{\mathbb{R}^{2}}(\xi)$ is a solution of the equation (we refer the reader to [2] for a detailed treatment of the subject)

$$
\begin{cases}\operatorname{Curl} \beta_{\mathbb{R}^{2}}(\xi)=\xi \delta_{0} & \text { in } \mathbb{R}^{2} \\ \operatorname{Div} \mathbb{C} \beta_{\mathbb{R}^{2}}(\xi)=0 & \text { in } \mathbb{R}^{2}\end{cases}
$$

Remark 4. As in Remark 3 make the annulus $C_{\varepsilon}$ a simply connected domain by introducing a cut with a segment $L$, and denote it by $\tilde{C}_{\varepsilon}:=C_{\varepsilon} \backslash L$ so that $\beta_{\mathbb{R}^{2}}(\xi)=\nabla u$ for some $u \in H^{1}\left(\tilde{C}_{\varepsilon} ; \mathbb{R}^{2}\right)$. Then by $[30$ it is very easy to prove that $u$ solves the following minimum problem:

$$
\begin{aligned}
& \min _{v, M, m}\left\{\int_{\tilde{C}_{\varepsilon}} \mathbb{C} \nabla v: \nabla v \mid v \in H^{1}\left(\tilde{C}_{\varepsilon}\right), v-u \in H^{1}\left(C_{\varepsilon}\right),\right. \\
&\left.v(x)=u(x) \text { on } \partial B_{1} \backslash L, v(x)=u(x)+M x+m \text { on } \partial B_{\varepsilon} \backslash L\right\},
\end{aligned}
$$


where $M$ is a skew matrix and $m \in \mathbb{R}^{2}$. Indeed, let $v$ be a minimizer; then $\nabla v$ solves the Euler-Lagrange equation

$$
\int_{C_{\varepsilon}} \mathbb{C} \nabla v: \nabla \varphi=0
$$

for every test function $\varphi$ such that $\varphi \in H^{1}\left(C_{\varepsilon}\right), \varphi(x)=0$ on $\partial B_{1}$, and $\varphi(x)=M x+m$ on $\partial B_{\varepsilon}$ for some skew matrix $M$ and $m \in \mathbb{R}^{2}$. Moreover, extending $\varphi$ in $B_{\varepsilon}$ by setting $\varphi=M x+m$ in $B_{\varepsilon}$, by we have

$$
0=\int_{B_{1}} \mathbb{C} \beta_{\mathbb{R}^{2}}(\xi): \nabla \varphi=\int_{C_{\varepsilon}} \mathbb{C} \beta_{\mathbb{R}^{2}}(\xi): \nabla \varphi,
$$

where in the last equality we have used that the scalar product between the symmetric matrix $\mathbb{C} \beta_{\mathbb{R}^{2}}(\xi)$ and the skew matrix $M$ vanishes. Finally, by (32) and (33), choosing $\varphi:=u-v$ we deduce

$$
\int_{C_{\varepsilon}} \mathbb{C} \nabla(u-v): \nabla(u-v)=0,
$$

which guarantees that $\nabla(u-v)$ is a skew matrix, so that $u$ minimizes 31.

In the next lemma we will see that in the cell formula (27) we can assign suitable boundary conditions without affecting the asymptotic behavior of the energy $\psi_{\varepsilon}(\xi)$. As a consequence, we will deduce that, up to small errors, we can plug the function $\beta_{\mathbb{R}^{2}}$ in the minimum problem 27].

Lemma 5. Let $\xi \in \mathbb{R}^{2}$ be fixed and let $\hat{\beta} \in \mathcal{A} \mathcal{S}_{1, \varepsilon}(\xi)$. Assume that

$$
|\hat{\beta}(x)| \leq \frac{K|\xi|}{|x|} \quad \text { for every } x \in C_{\varepsilon} \text { and for some } K>0 .
$$

Then there exists a function $\tilde{\beta} \in \mathcal{A} \mathcal{S}_{1, \varepsilon}(\xi)$ and a skew matrix $A$ such that

- $\tilde{\beta}$ coincides with $\hat{\beta}$ in a neighborhood of $\partial B_{1}$;

- $\tilde{\beta}$ coincides with $\hat{\beta}+A$ in a neighborhood of $\partial B_{\varepsilon}$;

- $\int_{C_{\varepsilon}} W(\tilde{\beta}) d x \leq \int_{C_{\varepsilon}} W\left(\beta_{\varepsilon}(\xi)\right) d x+C|\xi|^{2}$, where $C>0$ depends only on $K$.

Proof. As in Remark 3 , we make the annulus $C_{\varepsilon}$ a simply connected domain by introducing a cut with a segment $L$ and we denote it by $\tilde{C}_{\varepsilon}:=C_{\varepsilon} \backslash L$. Since both $\hat{\beta}$ and $\beta_{\varepsilon}(\xi)$ are curl free in $\tilde{C}_{\varepsilon}$, we have

$$
\beta_{\varepsilon}(\xi)=\nabla u, \quad \hat{\beta}=\nabla v \quad \text { for some } u, v \in H^{1}\left(\tilde{C}_{\varepsilon} ; \mathbb{R}^{2}\right) .
$$

Consider the annular sets $A_{n}:=B_{2^{n+1} \varepsilon} \backslash B_{2^{n} \varepsilon}, n \in \mathbb{N}$, which are well contained in $C_{\varepsilon}$. We can assume that there are at least two annular sets where the elastic energy of $u$ is not larger than the elastic energy of $v$, since otherwise, in view of (34), the statement would be trivially true with $A=0$ and $\tilde{\beta}=\hat{\beta}$. Let then $n_{1}$ and $n_{2}$ be the minimal and the maximal natural numbers such that the sets $A_{n_{i}}$ are contained in $C_{\varepsilon}$, and

$$
\int_{A_{n_{i}}} \mathbb{C} \nabla u: \nabla u \leq \int_{A_{n_{i}}} \mathbb{C} \nabla v: \nabla v \leq C|\xi|^{2}, \quad i \in\{1,2\} .
$$


By the Poincaré and Korn inequalities, there exist a positive constant $C>0$, two vectors $m_{1}, m_{2}$ and two skew matrices $M_{1}, M_{2}$ such that

$$
\begin{gathered}
\int_{A_{n_{i}}}\left|\nabla u-M_{i}\right|^{2} \leq C|\xi|^{2} \\
\int_{A_{n_{i}}}\left|u-M_{i} x-m_{i}\right|^{2} \leq C\left(2^{n_{i}} \varepsilon\right)^{2} \int_{A_{n_{i}}}\left|\nabla u-M_{i}\right|^{2} \leq C\left(2^{n_{i}} \varepsilon\right)^{2}|\xi|^{2} .
\end{gathered}
$$

Moreover, again by the Poincaré inequality there exist $l_{1}, l_{2} \in \mathbb{R}^{2}$ such that

$$
\int_{A_{n_{i}}}\left|v-l_{i}\right|^{2} \leq C\left(2^{n_{i}} \varepsilon\right)^{2}|\xi|^{2}
$$

Let $\varphi_{i}:\left(2^{n_{i}} \varepsilon, 2^{n_{i}+1} \varepsilon\right) \rightarrow \mathbb{R}$ be the affine function defined by the conditions $\varphi_{i}\left(2^{n_{i}} \varepsilon\right)$ $=0, \varphi_{i}\left(2^{n_{i}+1} \varepsilon\right)=1$, and set

$$
\tilde{\beta}(x):=\left\{\begin{array}{lc}
M_{1}-M_{2}+\hat{\beta} & \text { for }|x| \leq 2^{n_{1}} \varepsilon \\
M_{1}-M_{2}+\nabla\left(\varphi_{1}(|x|)\left(u(x)-M_{1} x-m_{1}\right)+\left(1-\varphi_{1}(|x|)\right)\left(v(x)-l_{1}\right)\right) & \text { for } x \in A_{n_{1}}, \\
-M_{2}+\beta_{\varepsilon}(\xi) & \text { for } 2^{n_{1}+1} \varepsilon \leq|x| \leq 2^{n_{2}} \varepsilon \\
\nabla\left(\varphi_{2}(|x|)\left(v(x)-l_{2}\right)+\left(1-\varphi_{2}(|x|)\right)\left(u(x)-M_{2} x-m_{2}\right)\right) & \text { for } 2^{n_{2}} \varepsilon \leq|x| \leq 2^{n_{2}+1} \varepsilon, \\
\hat{\beta} & \text { for }|x| \geq 2^{n_{2}+1} \varepsilon .
\end{array}\right.
$$

It is easy to check that $\tilde{\beta}$ belongs to $\mathcal{A} \mathcal{S}_{1, \varepsilon}(\xi)$ and satisfies all the desired conditions with $A=M_{1}-M_{2}$.

From Lemma 5, together with Remark 4 and 29], we deduce the following corollary.

Corollary 6. There exists a constant $C>0$ such that for every $\xi \in \mathbb{R}^{2}$,

$$
\psi_{\varepsilon}(\xi) \leq \frac{1}{|\log \varepsilon|} \int_{C_{\varepsilon}} W\left(\beta_{\mathbb{R}^{2}}(\xi)\right) d x \leq \psi_{\varepsilon}(\xi)+\frac{C|\xi|^{2}}{|\log \varepsilon|} .
$$

In particular, as $\varepsilon \rightarrow 0$ the functions $\psi_{\varepsilon}$ converge pointwise to the function $\psi: \mathbb{R}^{2} \rightarrow \mathbb{R}$, defined by

$$
\psi(\xi):=\lim _{\varepsilon \rightarrow 0} \psi_{\varepsilon}(\xi)=\frac{1}{|\log \varepsilon|} \lim _{\varepsilon \rightarrow 0} \int_{C_{\varepsilon}} W\left(\beta_{\mathbb{R}^{2}}(\xi)\right) d x=\int_{\partial B_{1}(0)} W\left(\Gamma_{\xi}(\theta)\right) d \theta .
$$

More precisely, we have

$$
\left|\psi_{\varepsilon}(\xi)-\psi(\xi)\right| \leq \frac{C|\xi|^{2}}{|\log \varepsilon|}
$$


Remark 7. In Corollary 6 we have proved that the field $\beta_{\mathbb{R}^{2}}(\xi)$ minimizes the cell problem in 27) up to errors killed by the logarithmic rescaling, and therefore the definition of $\psi(\xi)$ in (36) reduces to the line integral $\psi(\xi)=\int_{\partial B_{1}(0)} W\left(\Gamma_{\xi}(\theta)\right) d \theta$. We easily deduce the following variational characterization for the function $\psi$ in 36 :

$$
\begin{array}{r}
\psi(\xi):=\min \left\{\int_{\partial B_{1}} W(\Gamma(\theta)) d \theta \mid \Gamma: \partial B_{1} \rightarrow \mathbb{M}^{2 \times 2}, \operatorname{Curl} \frac{1}{r} \Gamma(\theta)=0,\right. \\
\\
\left.\qquad \int_{\partial B_{1}} \Gamma(\theta) \cdot t(\theta) d \theta=\xi\right\},
\end{array}
$$

where $t(\theta)$ denotes the tangent vector to $\partial B_{1}$. This is indeed also a variational characterization for the minimizer $\Gamma_{\xi}$, which is unique up to rotations (i.e., translations in $\theta$ ) and additive skew matrices.

By means of a simple change of variable $\varepsilon \rightarrow \rho$ we see that the self-energy is indeed concentrated in a $\rho$-neighborhood of the dislocation points whenever $|\log \rho| \ll|\log \varepsilon|$. The precise statement is given in the next proposition.

Proposition 8. For every $\varepsilon>0$ let $\rho_{\varepsilon}>0$ be such that $\log \rho_{\varepsilon} / \log \varepsilon \rightarrow 0$ as $\varepsilon \rightarrow 0$. Let $\bar{\psi}_{\varepsilon}: \mathbb{R}^{2} \rightarrow \mathbb{R}$ be defined through the following minimum problem:

$$
\bar{\psi}_{\varepsilon}(\xi):=\frac{1}{|\log \varepsilon|} \min \left\{\int_{B_{\rho_{\varepsilon}} \backslash B_{\varepsilon}} W(\beta) d x: \beta \in \mathcal{A} \mathcal{S}_{\varepsilon, \rho_{\varepsilon}}(\xi)\right\}
$$

Then $\bar{\psi}_{\varepsilon}=\psi_{\varepsilon}(1+o(\varepsilon))$, where $o(\varepsilon) \rightarrow 0$ as $\varepsilon \rightarrow 0$ uniformly with respect to $\xi$. In particular, $\bar{\psi}_{\varepsilon}$ converge pointwise as $\varepsilon \rightarrow 0$ to the function $\psi: \mathbb{R}^{2} \rightarrow \mathbb{R}$ given in Corollary 6.

Moreover, let $\hat{\beta} \in \mathcal{A} \mathcal{S}_{\varepsilon, \rho_{\varepsilon}}(\xi), \xi \in \mathbb{R}^{2}$, be such that

$$
|\hat{\beta}(x)| \leq K \frac{|\xi|}{|x|}
$$

for some $K \in \mathbb{R}$, and let $\tilde{\psi}_{\varepsilon}: \mathbb{R}^{2} \rightarrow \mathbb{R}$ be defined through the following minimum problem:

$$
\tilde{\psi}_{\varepsilon}(\xi):=\frac{1}{|\log \varepsilon|} \min \left\{\int_{B_{\rho_{\varepsilon}} \backslash B_{\varepsilon}} W(\beta) d x: \beta \in \mathcal{A} \mathcal{S}_{\varepsilon, \rho_{\varepsilon}}(\xi), \beta \cdot t=\hat{\beta} \cdot \text { t on } \partial B_{\varepsilon} \cup \partial B_{\rho_{\varepsilon}}\right\} \text {. }
$$

Then $\tilde{\psi}_{\varepsilon}=\psi_{\varepsilon}(1+o(\varepsilon))$, where $o(\varepsilon) \rightarrow 0$ as $\varepsilon \rightarrow 0$ uniformly with respect to $\xi$. In particular, $\tilde{\psi}_{\varepsilon}$ converge pointwise as $\varepsilon \rightarrow 0$ to the function $\psi: \mathbb{R}^{2} \rightarrow \mathbb{R}$ given in Corollary 6 .

Remark 9. The error $o(\varepsilon)$ appearing in the expression of $\bar{\psi}$ and $\tilde{\psi}$ in Proposition 8 can be estimated as follows: $o(\varepsilon) \approx \log \rho_{\varepsilon} / \log \varepsilon$. 
We are now in a position to define the density $\varphi: \mathbb{R}^{2} \rightarrow[0, \infty)$ of the self-energy through the following relaxation procedure:

$$
\varphi(\xi):=\inf \left\{\sum_{k=1}^{N} \lambda_{k} \psi\left(\xi_{k}\right): \sum_{k=1}^{N} \lambda_{k} \xi_{k}=\xi, N \in \mathbb{N}, \lambda_{k} \geq 0, \xi_{k} \in \mathbb{S}\right\} .
$$

It follows from the definition that the function $\varphi$ is positively 1-homogeneous and convex. Moreover, since $\psi(\xi) \geq C|\xi|^{2}$ for some $C \geq 0$ (which can be checked by the very definition), the inf in (42) is indeed a minimum.

Remark 10. Note that if for every $z_{1}, \ldots, z_{s} \in \mathbb{Z}$ we have

$$
\psi\left(\sum_{i=1}^{s} z_{i} b_{i}\right) \geq \sum_{i=1}^{s} z_{i} \psi\left(b_{i}\right)
$$

then in the relaxation procedure given in 442 we can replace $\mathbb{S}$ with $S$. More precisely, the formula for $\varphi$ reduces to

$$
\varphi(\xi):=\min \left\{\sum_{i=1}^{s}\left|\lambda_{i}\right| \psi\left(b_{i}\right): \sum_{i=1}^{s} \lambda_{i} b_{i}=\xi, b_{i} \in S\right\} .
$$

Actually, condition (43) can be viewed as a condition that the class of Burgers vectors $b_{i}$ of the given crystal has to satisfy in order to contain all the dislocation's defects observed in the crystal. In other words, if a dislocation corresponding to a vector $b:=\sum_{i=1}^{s} z_{i} b_{i}$ stores an energy smaller than that obtained by separating all its components $b_{i}$, then $b$ itself has to be considered as a Burgers vector of the crystal. A rigorous mathematical definition of the class of Burgers vectors corresponding to a given crystal could be to consider the set $\left\{b_{1}, \ldots, b_{s}\right\} \subset \mathbb{S}$ of all vectors satisfying $\psi\left(b_{i}\right)=\varphi\left(b_{i}\right)$, where $\mathbb{S}$ is the set of slips under which the crystal is invariant. The Burgers vectors defined in such a way would always satisfy property (43).

\section{A Korn type inequality for fields with prescribed curl}

Let $u \in W^{1,2}\left(\Omega ; \mathbb{R}^{2}\right), \beta$ its gradient and $\beta^{\text {sym }}$ and $\beta^{\text {skew }}$ its decomposition into symmetric and anti-symmetric parts. The classical Korn inequality asserts that if $\beta^{\text {skew }}$ has zero mean value, then its $L^{2}$ norm is controlled by the $L^{2}$ norm of $\beta^{\text {sym }}$. We will show that in dimension two the same result holds true also for fields $\beta$ that are not curl free, modulo an error depending actually on the mass of $\operatorname{Curl} \beta$. The result is a consequence of some estimates for elliptic systems with $L^{1}$ data recently proved by Bourgain, Brezis and Van Schaftingen in [3], [5]. The precise statement is the following

Theorem 11 (A generalized Korn type inequality). There exists a constant $C$ depending only on $\Omega$ such that for every $\beta \in L^{1}\left(\Omega ; \mathbb{M}^{2 \times 2}\right)$ with

$$
\operatorname{Curl} \beta=\mu \in \mathcal{M}\left(\Omega ; \mathbb{R}^{2}\right), \quad \int_{\Omega} \beta^{\text {skew }}=0,
$$


we have

$$
\int_{\Omega}\left|\beta^{\text {skew }}\right|^{2} d x \leq C\left(\int_{\Omega}\left|\beta^{\text {sym }}\right|^{2} d x+(|\mu|(\Omega))^{2}\right) .
$$

Proof. The condition $\operatorname{Curl} \beta=\left(\mu_{1}, \mu_{2}\right)$ can be written in the form

$$
\left\{\begin{array}{l}
\left(\beta_{12}^{\text {skew }}\right)_{x_{1}}=h_{1}+\mu_{1} \\
\left(\beta_{12}^{\text {skew }}\right)_{x_{2}}=h_{2}+\mu_{2}
\end{array}\right.
$$

where $h_{i} \in H^{-1}(\Omega)$ are linear combinations of derivatives of entries of $\beta^{\text {sym }}$.

Since the field $\left(\left(\beta_{12}^{\text {skew }}\right)_{x_{1}},\left(\beta_{12}^{\text {skew }}\right)_{x_{2}}\right)$ is curl free, we deduce that $\operatorname{curl}\left(\mu_{1}, \mu_{2}\right)=$ $\operatorname{curl}\left(-h_{1},-h_{2}\right)$, or equivalently

$$
\operatorname{div}\left(-\mu_{2}, \mu_{1}\right)=\operatorname{div}\left(h_{2},-h_{1}\right) .
$$

By [5, Theorem 3.1 and Remark 3.3] (see also [25] and [3]), if $f \in L^{1}\left(\Omega ; \mathbb{R}^{2}\right)$ is a vector field satisfying $\operatorname{div} f \in H^{-2}(\Omega)$, then $f$ also belongs to $H^{-1}\left(\Omega ; \mathbb{R}^{2}\right)$ and the following estimate holds:

$$
\|f\|_{H^{-1}\left(\Omega ; \mathbb{R}^{2}\right)} \leq c\left(\|\operatorname{div} f\|_{H^{-2}(\Omega)}+\|f\|_{L^{1}\left(\Omega ; \mathbb{R}^{2}\right)}\right) .
$$

This result clearly extends by density to measures with bounded variation. Thus, by (46), we have $\operatorname{div}\left(-\mu_{2}, \mu_{1}\right) \in H^{-2}(\Omega)$, and so we deduce that $\mu$ belongs to $H^{-1}\left(\Omega ; \mathbb{R}^{2}\right)$ and

$$
\|\mu\|_{H^{-1}\left(\Omega ; \mathbb{R}^{2}\right)} \leq c\left(\left\|\operatorname{div}\left(h_{2},-h_{1}\right)\right\|_{H^{-2}(\Omega)}+|\mu|(\Omega)\right) \leq c\left(\left\|\beta^{\mathrm{sym}}\right\|_{L^{2}\left(\Omega ; \mathbb{R}^{2}\right)}+|\mu|(\Omega)\right) .
$$

Now let $u \in H_{0}^{1}\left(\Omega ; \mathbb{R}^{2}\right)$ be the solution of $-\Delta u=\left(-\mu_{2}, \mu_{1}\right)$ in $\Omega$ and let $\xi$ be the $2 \times 2$ matrix defined by $\xi:=J \nabla u$ (i.e., the $i^{\text {th }}$ row of $\xi$ is given by $\xi_{i}=\left(-\left(u_{i}\right)_{x_{2}},\left(u_{i}\right)_{x_{1}}\right)$, for $i=1,2$ ). By definition we have $\operatorname{Curl} \xi=\mu$. By (47) we then obtain

$$
\int_{\Omega}|\xi|^{2} d x=\|\nabla u\|_{L^{2}}^{2} \leq c\|\mu\|_{H^{-1}\left(\Omega ; \mathbb{R}^{2}\right)}^{2} \leq\left(\int_{\Omega}\left|\beta^{\mathrm{sym}}\right|^{2} d x+(|\mu|(\Omega))^{2}\right) .
$$

Since the average of the anti-symmetric part of $\xi$ can be easily estimated by the $L^{2}$ norm of $\xi$, we can assume that 48 holds for a matrix $\xi$ such that $\xi^{\text {skew }}$ has zero mean value and $\operatorname{Curl} \xi=\mu$. Therefore, by the classical Korn inequality applied to $\beta-\xi$, which by construction is curl free, and in view of (48) we conclude

$$
\begin{aligned}
\int_{\Omega}\left|\beta^{\text {skew }}\right|^{2} d x & \leq c\left(\int_{\Omega}\left|\beta^{\text {skew }}-\xi^{\text {skew }}\right|^{2} d x+\int_{\Omega}\left|\xi^{\text {skew }}\right|^{2} d x\right) \\
& \leq c\left(\int_{\Omega}\left|\beta^{\text {sym }}-\xi^{\text {sym }}\right|^{2} d x+\int_{\Omega}\left|\xi^{\text {skew }}\right|^{2} d x\right) \\
& \leq c\left(\int_{\Omega}\left|\beta^{\text {sym }}\right|^{2} d x+\int_{\Omega}|\xi|^{2} d x\right) \\
& \leq c\left(\int_{\Omega}\left|\beta^{\text {sym }}\right|^{2} d x+(|\mu|(\Omega))^{2}\right)
\end{aligned}
$$




\section{The critical regime $\left(N_{\varepsilon} \approx|\log \varepsilon|\right)$}

In this section we will study the asymptotic behavior of the rescaled energy functionals as the internal scale $\varepsilon \rightarrow 0$, in the critical energy regime, namely with $N_{\varepsilon}=|\log \varepsilon|$. In terms of $\Gamma$-convergence, we consider the rescaled energy functionals $\mathcal{F}_{\varepsilon}: \mathcal{M}\left(\Omega ; \mathbb{R}^{2}\right) \times$ $L^{1}\left(\Omega ; \mathbb{M}^{2 \times 2}\right) \rightarrow \mathbb{R}$ defined in 22 .

According to the heuristic arguments above, in this regime we expect the coexistence of the two effects, the interaction energy and the self-energy, so that the candidate for the $\Gamma$-limit $\mathcal{F}: \mathcal{M}\left(\Omega ; \mathbb{R}^{2}\right) \times L^{2}\left(\Omega ; \mathbb{M}^{2 \times 2}\right) \rightarrow \mathbb{R}$ is defined by

$$
\mathcal{F}(\mu, \beta):= \begin{cases}\int_{\Omega} W(\beta) d x+\int_{\Omega} \varphi\left(\frac{d \mu}{d|\mu|}\right) d|\mu| & \text { if } \mu \in H^{-1}\left(\Omega ; \mathbb{R}^{2}\right), \text { Curl } \beta=\mu \\ \infty & \text { otherwise in } L^{2}\left(\Omega ; \mathbb{M}^{2 \times 2}\right)\end{cases}
$$

Theorem 12. The following $\Gamma$-convergence result holds.

(i) (Compactness) Let $\varepsilon_{n} \rightarrow 0$ and let $\left\{\left(\mu_{n}, \beta_{n}\right)\right\}$ be a sequence in $\mathcal{M}\left(\Omega ; \mathbb{R}^{2}\right) \times$ $L^{2}\left(\Omega ; \mathbb{M}^{2 \times 2}\right)$ such that $\mathcal{F}_{\varepsilon_{n}}\left(\mu_{n}, \beta_{n}\right) \leq E$ for some positive constant $E$ independent of $n$. Then there exist a subsequence of $\varepsilon_{n}$ (not relabeled), a measure $\mu \in$ $H^{-1}\left(\Omega ; \mathbb{R}^{2}\right)$, and a strain $\beta \in L^{2}\left(\Omega ; \mathbb{M}^{2 \times 2}\right)$, with $\operatorname{Curl} \beta=\mu$, such that

$$
\begin{aligned}
& \frac{1}{\left|\log \varepsilon_{n}\right|} \mu_{n} \stackrel{*}{\rightarrow} \mu \quad \text { in } \mathcal{M}\left(\Omega ; \mathbb{R}^{2}\right), \\
& \frac{1}{\left|\log \varepsilon_{n}\right|} \beta_{n} \rightarrow \beta \quad \text { in } L^{2}\left(\Omega ; \mathbb{M}^{2 \times 2}\right) .
\end{aligned}
$$

(ii) ( $\Gamma$-convergence) The functionals $\mathcal{F}_{\varepsilon} \Gamma$-converge to $\mathcal{F}$ as $\varepsilon \rightarrow 0$ with respect to the convergence in (50), 51, i.e., the following inequalities hold:

- $\left(\Gamma\right.$-liminf inequality) for $(\mu, \beta) \in\left(\mathcal{M}\left(\Omega ; \mathbb{R}^{2}\right) \cap H^{-1}\left(\Omega ; \mathbb{R}^{2}\right)\right) \times L^{2}\left(\Omega ; \mathbb{M}^{2 \times 2}\right)$ with $\operatorname{Curl} \beta=\mu$ and for every sequence $\left(\mu_{\varepsilon}, \beta_{\varepsilon}\right) \in X_{\varepsilon} \times L^{2}\left(\Omega ; \mathbb{M}^{2 \times 2}\right)$ satisfying 50) and (51), we have

$$
\liminf _{\varepsilon \rightarrow 0} \mathcal{F}_{\varepsilon}\left(\mu_{\varepsilon}, \beta_{\varepsilon}\right) \geq \mathcal{F}(\mu, \beta)
$$

- $\left(\Gamma\right.$-limsup inequality) given $(\mu, \beta) \in\left(\mathcal{M}\left(\Omega ; \mathbb{R}^{2}\right) \cap H^{-1}\left(\Omega ; \mathbb{R}^{2}\right)\right) \times L^{2}\left(\Omega ; \mathbb{M}^{2 \times 2}\right)$ with $\operatorname{Curl} \beta=\mu$, there exists $\left(\mu_{\varepsilon}, \beta_{\varepsilon}\right) \in \mathcal{M}\left(\Omega ; \mathbb{R}^{2}\right) \times L^{2}\left(\Omega ; \mathbb{M}^{2 \times 2}\right)$ satisfying (50) and (51) such that

$$
\limsup _{\varepsilon \rightarrow 0} \mathcal{F}_{\varepsilon}\left(\mu_{\varepsilon}, \beta_{\varepsilon}\right) \leq \mathcal{F}(\mu, \beta) .
$$

Remark 13. Consider the functionals $\mathcal{E}_{\varepsilon}(\mu):=\min _{\beta} \mathcal{F}_{\varepsilon}(\mu, \beta)$, representing the elastic energy induced by the dislocation measure $\mu$. By the $\Gamma$-convergence result stated in Theorem 12 we immediately deduce that the functionals $\mathcal{E}_{\varepsilon} \Gamma$-converge (as $\varepsilon \rightarrow 0$ ) to the functional $\mathcal{E}: \mathcal{M}\left(\Omega ; \mathbb{R}^{2}\right) \cap H^{-1}\left(\Omega ; \mathbb{R}^{2}\right) \rightarrow \mathbb{R}$ defined by

$$
\mathcal{E}(\mu):=\min _{\beta}\{\mathcal{F}(\mu, \beta): \operatorname{Curl} \beta=\mu\} .
$$


Therefore, the energy $\mathcal{E}(\mu)$ induced by a distribution of dislocations $\mu$ in the critical regime is given by the sum of both an elastic and a plastic term. In particular, any distribution of dislocations in this regime induces a residual elastic distortion (i.e., if $\mu$ is not zero, then so is the corresponding strain $\beta$ that minimizes $[52]$ and hence its elastic energy).

\subsection{Compactness}

Let $\left\{\left(\mu_{n}, \beta_{n}\right)\right\}$ be a sequence in $\mathcal{M}\left(\Omega ; \mathbb{R}^{2}\right) \times L^{2}\left(\Omega ; \mathbb{M}^{2 \times 2}\right)$ such that $\mathcal{F}_{\varepsilon_{n}}\left(\mu_{n}, \beta_{n}\right) \leq E$ for some positive constant $E$ independent of $n$. We give the proof of the compactness property stated in Theorem 12 in three steps.

Step 1. Weak compactness of the rescaled dislocation measures.

We first show that the sequence $\left\{\left(1 /\left|\log \varepsilon_{n}\right|\right) \mu_{n}\right\}$ is uniformly bounded in mass. Let $\mu_{n}=$ $\sum_{i=1}^{M_{n}} \xi_{i, n} \delta_{x_{i, n}}$ with $\xi_{i, n} \in \mathbb{S}$; we claim that

$$
\frac{1}{\left|\log \varepsilon_{n}\right|}\left|\mu_{n}\right|(\Omega)=\frac{1}{\left|\log \varepsilon_{n}\right|} \sum_{i=1}^{M_{n}}\left|\xi_{i, n}\right| \leq C .
$$

Indeed,

$$
E \geq \mathcal{F}_{\varepsilon_{n}}\left(\mu_{n}, \beta_{n}\right)=\frac{1}{\left|\log \varepsilon_{n}\right|^{2}} \int_{\Omega_{\varepsilon}\left(\mu_{n}\right)} W\left(\beta_{n}\right) d x \geq \sum_{i=1}^{M_{n}} \frac{1}{\left|\log \varepsilon_{n}\right|^{2}} \int_{B_{\rho_{\varepsilon_{n}}}\left(x_{i, n}\right)} W\left(\beta_{n}\right) d x,
$$

where we recall that $\beta_{n}=0$ in $\Omega \backslash \Omega_{\varepsilon}\left(\mu_{n}\right)$. After a change of variables we deduce

$$
E \geq \sum_{i=1}^{M_{n}} \frac{1}{\left|\log \varepsilon_{n}\right|^{2}} \int_{B_{\rho_{\varepsilon_{n}}}} W\left(\beta_{n}\left(x_{i, n}+y\right)\right) d y .
$$

Note that the functions $y \mapsto \beta_{n}\left(x_{i, n}+y\right)$ belong to the class $\mathcal{A} \mathcal{S}_{\varepsilon_{n}, \rho_{\varepsilon_{n}}}\left(\xi_{i, n}\right)$ defined in 26). Therefore we have

$$
\begin{aligned}
E & \geq \sum_{i=1}^{M_{n}} \frac{1}{\left|\log \varepsilon_{n}\right|^{2}} \int_{B_{\rho_{\varepsilon_{n}}}} W\left(\beta_{n}\left(x_{i, n}+y\right)\right) d y \\
& \geq \frac{1}{\left|\log \varepsilon_{n}\right|} \sum_{i=1}^{M_{n}} \bar{\psi}_{\varepsilon_{n}}\left(\xi_{i, n}\right)=\frac{1}{\left|\log \varepsilon_{n}\right|} \sum_{i=1}^{M_{n}}\left|\xi_{i, n}\right|^{2} \bar{\psi}_{\varepsilon_{n}}\left(\frac{\xi_{i, n}}{\left|\xi_{i, n}\right|}\right),
\end{aligned}
$$

where the function $\bar{\psi}_{\varepsilon_{n}}$ is defined in 39 . Let $\psi$ be the function given by Corollary 6 , and let $2 c:=\inf _{|\xi|=1} \psi(\xi)$. By Proposition 8 we deduce that for $n$ large enough $\bar{\psi}_{\varepsilon_{n}}(\xi) \geq c$ for every $\xi$ with $|\xi|=1$. By [54] we obtain

$$
E \geq \frac{1}{\left|\log \varepsilon_{n}\right|} \sum_{i=1}^{M_{n}}\left|\xi_{i, n}\right|^{2} \bar{\psi}_{\varepsilon_{n}}\left(\frac{\xi_{i, n}}{\left|\xi_{i, n}\right|}\right) \geq \frac{c}{\left|\log \varepsilon_{n}\right|} \sum_{i=1}^{M_{n}}\left|\xi_{i, n}\right|^{2} \geq \frac{C}{\left|\log \varepsilon_{n}\right|} \sum_{i=1}^{M_{n}}\left|\xi_{i, n}\right|,
$$


where the last inequality follows from the fact that $\xi_{i, n} \in \mathbb{S}=\operatorname{Span}_{\mathbb{Z}} S, S$ is a finite set, and hence $\left|\xi_{i, n}\right|$ are bounded away from zero. We conclude that (53) holds.

Step 2. Weak compactness of the rescaled strains.

In view of the coercivity condition (3) we have

$$
C\left|\log \varepsilon_{n}\right|^{2} \geq C\left|\log \varepsilon_{n}\right|^{2} \mathcal{F}_{\varepsilon}\left(\mu_{n}, \beta_{n}\right) \geq C \int_{\Omega} W\left(\beta_{n}\right) d x \geq \int_{\Omega}\left|\beta_{n}^{\text {sym }}\right|^{2} d x .
$$

The idea of the proof is to apply the generalized Korn inequality provided by Theorem 11 to $\beta_{n}$ to control the anti-symmetric part of $\beta_{n}$. Note that the curl of $\beta_{n}$ is clearly related to the dislocation measure $\mu_{n}$, whose mass is bounded by $C\left|\log \varepsilon_{n}\right|$ by Step 1. On the other hand, it is not clear that $\left|\operatorname{Curl} \beta_{n}\right| \leq C\left|\log \varepsilon_{n}\right|$. Therefore we proceed as follows. For every $x_{i, n}$ in the support set of $\mu_{n}$, set $C_{i, n}:=B_{2 \varepsilon_{n}}\left(x_{i, n}\right) \backslash B_{\varepsilon_{n}}\left(x_{i, n}\right)$ and consider the function $K_{i, n}: C_{i, n} \rightarrow \mathbb{M}^{2 \times 2}$ defined by

$$
K_{i, n}:=\frac{1}{2 \pi} \xi_{i, n} \otimes J \frac{x-x_{i}}{\left|x-x_{i}\right|^{2}},
$$

where $J$ is the clockwise rotation of $90^{\circ}$. It is easy to show that

$$
\int_{C_{i, n}}\left|K_{i, n}\right|^{2} d x \leq c \int_{C_{i, n}}\left|\beta_{n}^{\mathrm{sym}}\right|^{2} d x .
$$

Indeed, it is straightforward to check that

$$
\int_{C_{i, n}}\left|K_{i, n}\right|^{2} d x=C\left|\xi_{i, n}\right|^{2}
$$

and by a scaling argument, from Remark 3 we get

$$
\int_{C_{i, n}}\left|\beta_{n}^{\mathrm{sym}}\right|^{2} d x \geq c\left|\xi_{i, n}\right|^{2}
$$

and hence $(56)$.

By construction $\operatorname{Curl}\left(\beta_{n}-K_{i, n}\right)=0$ in $C_{i, n}$, and so $\beta_{n}-K_{i, n}=\nabla v_{i, n}$ in $C_{i, n}$ for some $v_{i, n} \in H^{1}\left(C_{i, n} ; \mathbb{R}^{2}\right)$. Thus by 56 , we have

$$
\int_{C_{i, n}}\left|\nabla v_{i, n}^{\mathrm{sym}}\right|^{2} d x \leq C \int_{C_{i, n}}\left(\left|\beta_{n}^{\mathrm{sym}}\right|^{2}+\left|K_{i, n}\right|^{2}\right) d x \leq C \int_{C_{i, n}}\left|\beta_{n}^{\mathrm{sym}}\right|^{2} d x,
$$

and hence, applying the standard Korn inequality to $v_{i, n}$, we deduce that

$$
\int_{C_{i, n}}\left|\nabla v_{i, n}-A_{i, n}\right|^{2} d x \leq C \int_{C_{i, n}}\left|\nabla v_{i, n}^{\mathrm{sym}}\right|^{2} d x \leq C \int_{C_{i, n}}\left|\beta_{n}^{\mathrm{sym}}\right|^{2} d x,
$$

where $A_{i, n}$ is a suitable anti-symmetric matrix. By standard extension arguments there exists a function $u_{i, n} \in H^{1}\left(B_{2 \varepsilon_{n}}\left(x_{i, n}\right) ; \mathbb{R}^{2}\right)$ such that $\nabla u_{i, n} \equiv \nabla v_{i, n}-A_{i, n}$ in $C_{i, n}$ and

$$
\int_{B_{2 \varepsilon_{n}}\left(x_{i, n}\right)}\left|\nabla u_{i, n}\right|^{2} d x \leq \int_{C_{i, n}}\left|\nabla v_{i, n}-A_{i, n}\right|^{2} d x \leq C \int_{C_{i, n}}\left|\beta_{n}^{\mathrm{sym}}\right|^{2} d x .
$$


Consider the field $\tilde{\beta}_{n}: \Omega \rightarrow \mathbb{M}^{2 \times 2}$ defined by

$$
\tilde{\beta}_{n}(x):= \begin{cases}\beta_{n}(x) & \text { if } x \in \Omega_{\varepsilon_{n}}, \\ \nabla u_{i, n}(x)+A_{i, n} & \text { if } x \in B_{\varepsilon_{n}}\left(x_{i, n}\right) .\end{cases}
$$

In view of (57) it follows that

$$
\begin{aligned}
\int_{\Omega}\left|\tilde{\beta}_{n}^{\mathrm{sym}}\right|^{2} d x & =\int_{\Omega}\left|\beta_{n}^{\mathrm{sym}}\right|^{2} d x+\sum_{i} \int_{B_{\varepsilon_{n}}\left(x_{i, n}\right)}\left|\nabla u_{i, n}^{\mathrm{sym}}\right|^{2} d x \leq C \int_{\Omega}\left|\beta_{n}^{\mathrm{sym}}\right|^{2} d x \\
& \leq C\left|\log \varepsilon_{n}\right|^{2} .
\end{aligned}
$$

By construction we have $\left|\operatorname{Curl} \tilde{\beta}_{n}\right|(\Omega)=\left|\mu_{n}\right|(\Omega)$; therefore, we can apply Theorem 11 to $\tilde{\beta}_{n}$, obtaining

$$
\int_{\Omega}\left|\tilde{\beta}_{n}-\tilde{A}_{n}\right|^{2} d x \leq C\left(\int_{\Omega}\left|\tilde{\beta}_{n}^{\text {sym }}\right|^{2} d x+\left(\left|\mu_{n}\right|(\Omega)\right)^{2}\right) \leq C\left|\log \varepsilon_{n}\right|^{2},
$$

where $\tilde{A}_{n}$ is the average of the anti-symmetric part of $\tilde{\beta}_{n}$. Since the average of $\beta_{n}$ is a symmetric matrix, we have

$$
\int_{\Omega_{\varepsilon_{n}}\left(\mu_{n}\right)}\left|\beta_{n}\right|^{2} d x=\int_{\Omega_{\varepsilon_{n}}\left(\mu_{n}\right)}\left(\left|\beta_{n}-\tilde{A}_{n}\right|^{2}-\left|\tilde{A}_{n}\right|^{2}\right) d x \leq \int_{\Omega_{\varepsilon_{n}}\left(\mu_{n}\right)}\left|\beta_{n}-\tilde{A}_{n}\right|^{2} d x .
$$

Finally, by 588 and $(59)$ we conclude

$$
\int_{\Omega_{\varepsilon_{n}}\left(\mu_{n}\right)}\left|\beta_{n}\right|^{2} d x \leq \int_{\Omega_{\varepsilon_{n}}\left(\mu_{n}\right)}\left|\beta_{n}-\tilde{A}_{n}\right|^{2} d x \leq \int_{\Omega}\left|\tilde{\beta}_{n}-\tilde{A}_{n}\right|^{2} d x \leq C\left|\log \varepsilon_{n}\right|^{2},
$$

which gives the desired compactness property for $\beta_{n} /\left|\log \varepsilon_{n}\right|$ in $L^{2}\left(\Omega ; \mathbb{M}^{2 \times 2}\right)$.

Step 3. $\mu$ belongs to $H^{-1}\left(\Omega ; \mathbb{R}^{2}\right)$ and $\operatorname{Curl} \beta=\mu$.

Let $\varphi \in C_{0}^{1}(\Omega)$. It is easy to construct a sequence $\left\{\varphi_{n}\right\} \subset H_{0}^{1}(\Omega)$ converging to $\varphi$ uniformly and strongly in $H_{0}^{1}(\Omega)$ and satisfying the property

$$
\varphi_{n} \equiv \varphi\left(x_{i, n}\right) \quad \text { in } B_{\varepsilon_{n}}\left(x_{i, n}\right) \quad \text { for every } x_{i, n} \text { in the support of } \mu_{n} .
$$

By Remark 1 we have

$$
\begin{aligned}
\int_{\Omega} \varphi d \mu & =\lim _{n \rightarrow+\infty} \frac{1}{\left|\log \varepsilon_{n}\right|} \int_{\Omega} \varphi_{n} d \mu_{n}=\lim _{n \rightarrow+\infty} \frac{1}{\left|\log \varepsilon_{n}\right|}\left\langle\operatorname{Curl} \beta_{n}, \varphi_{n}\right\rangle \\
& =\lim _{n \rightarrow+\infty} \frac{1}{\left|\log \varepsilon_{n}\right|} \int_{\Omega} \beta_{n} J \nabla \varphi_{n} d x=\int_{\Omega} \beta J \nabla \varphi d x=\langle\operatorname{Curl} \beta, \varphi\rangle,
\end{aligned}
$$

from which we deduce the admissibility condition $\operatorname{Curl} \beta=\mu$. Moreover, since by the previous step we have $\beta \in L^{2}\left(\Omega ; \mathbb{M}^{2 \times 2}\right)$, we deduce that $\mu$ belongs to $H^{-1}\left(\Omega ; \mathbb{R}^{2}\right)$. 


\section{2. $\Gamma$-liminf inequality}

Here we prove the $\Gamma$-liminf inequality of Theorem 12 . Let

$$
(\mu, \beta) \in \mathcal{M}\left(\Omega ; \mathbb{R}^{2}\right) \times L^{2}\left(\Omega ; \mathbb{M}^{2 \times 2}\right) \quad \text { with } \operatorname{Curl} \beta=\mu,
$$

and let $\left(\mu_{\varepsilon}, \beta_{\varepsilon}\right)$ satisfy $[50)$ and 51$]$. In order to prove the $\Gamma$-liminf inequality

$$
\liminf _{\varepsilon \rightarrow 0} \mathcal{F}_{\varepsilon}\left(\mu_{\varepsilon}, \beta_{\varepsilon}\right) \geq \mathcal{F}(\mu, \beta),
$$

it is enough to show that it holds for the self-energy and the interaction energy separately. More precisely, we write the energy corresponding to $\left(\mu_{\varepsilon}, \beta_{\varepsilon}\right)$ in the following way:

$$
\mathcal{F}_{\varepsilon}\left(\mu_{\varepsilon}, \beta_{\varepsilon}\right)=\int_{\Omega} \chi \cup B_{\rho_{\varepsilon}}\left(x_{i, \varepsilon}\right) W\left(\beta_{\varepsilon}\right) d x+\int_{\Omega} \chi_{\Omega \backslash \cup B_{\rho_{\varepsilon}}\left(x_{i, \varepsilon}\right)} W\left(\beta_{\varepsilon}\right) d x
$$

and we estimate the two terms separately. Set $\eta_{\varepsilon}:=\sum_{i=1}^{M_{\varepsilon}} \delta_{x_{i, \varepsilon}}$. By Proposition 8 we have

$$
\frac{1}{|\log \varepsilon|} \int_{\Omega} \chi \cup B_{\rho_{\varepsilon}}\left(x_{i, \varepsilon}\right) W\left(\beta_{n}\right) d x \geq \int_{\Omega} \bar{\psi}_{\varepsilon}\left(\frac{d \mu_{\varepsilon}}{d \eta_{\varepsilon}}\right) d \eta_{\varepsilon} \geq \int_{\Omega} \psi\left(\frac{d \mu_{\varepsilon}}{d \eta_{\varepsilon}}\right)(1+o(\varepsilon)) d \eta_{\varepsilon}
$$

where $o(\varepsilon) \rightarrow 0$ as $\varepsilon \rightarrow 0$. Since $\operatorname{Span}_{\mathbb{R}} \mathbb{S}=\mathbb{R}^{2}$, the convex 1-homogeneous function $\varphi$ defined in (42) is finite in $\mathbb{R}^{2}$, and so continuous. Thus, in view of Reshetnyak's Theorem (see [22, Theorem 1.2]), we deduce

$$
\begin{aligned}
\liminf _{\varepsilon \rightarrow 0} \frac{1}{|\log \varepsilon|} \int_{\Omega} \psi\left(\frac{d \mu_{\varepsilon}}{d \eta_{\varepsilon}}\right) d \eta_{\varepsilon} \geq \liminf _{\varepsilon \rightarrow 0} \frac{1}{|\log \varepsilon|} \int_{\Omega} \varphi\left(\frac{d \mu_{\varepsilon}}{d \eta_{\varepsilon}}\right) d \eta_{\varepsilon} \\
=\liminf _{\varepsilon \rightarrow 0} \frac{1}{|\log \varepsilon|} \int_{\Omega} \varphi\left(\frac{d \mu_{\varepsilon}}{d\left|\mu_{\varepsilon}\right|}\right) d\left|\mu_{\varepsilon}\right| \geq \int_{\Omega} \varphi\left(\frac{d \mu}{d|\mu|}\right) d|\mu| .
\end{aligned}
$$

From 61 and 62 we find that the $\Gamma$-liminf inequality holds for the self-energy, i.e.,

$$
\liminf _{\varepsilon \rightarrow 0} \frac{1}{|\log \varepsilon|^{2}} \int_{\Omega} \chi \cup B_{\rho_{\varepsilon}}\left(x_{i, \varepsilon}\right) W\left(\beta_{\varepsilon}\right) d x \geq \int_{\Omega} \varphi\left(\frac{d \mu}{d|\mu|}\right) d|\mu| .
$$

Concerning the interaction energy, by semicontinuity we immediately deduce

$$
\begin{aligned}
\liminf _{\varepsilon \rightarrow 0} & \frac{1}{|\log \varepsilon|^{2}} \int_{\Omega} \chi_{\Omega \backslash \bigcup B_{\rho_{\varepsilon}}\left(x_{i, \varepsilon}\right)} W\left(\beta_{\varepsilon}\right) d x \\
\quad & =\liminf _{\varepsilon \rightarrow 0} \int_{\Omega} W\left(\chi_{\Omega \backslash \cup B_{\rho_{\varepsilon}}\left(x_{i, \varepsilon}\right)} \frac{1}{|\log \varepsilon|} \beta_{\varepsilon}^{\mathrm{sym}}\right) d x \geq \int_{\Omega} W\left(\beta^{\mathrm{sym}}\right) d x .
\end{aligned}
$$

In the last inequality we used the fact that the number of atoms of $\mu_{\varepsilon}$ is bounded by $|\log \varepsilon|$ and hence, since by assumption $|\log \varepsilon| \rho_{\varepsilon}^{2} \rightarrow 0$, the function $\chi_{\Omega \backslash \bigcup B_{\rho_{\varepsilon}}\left(x_{i, \varepsilon}\right)}$ converges strongly to 1 .

Inequality $[63]$, together with inequality $\lfloor 64]$, gives the $\Gamma$-liminf inequality. 


\section{3. $\Gamma$-limsup inequality}

Here we prove the $\Gamma$-limsup inequality of Theorem 12 . We begin with a lemma that will be useful in the construction of recovery sequences also for different energy regimes under consideration. Given $\mu_{\varepsilon}:=\sum_{i=1}^{M_{\varepsilon}} \xi_{i, \varepsilon} \delta_{x_{i, \varepsilon}} \in X_{\varepsilon}$ and $r_{\varepsilon} \rightarrow 0$, we introduce the corresponding measures, diffused on balls of radius $r_{\varepsilon}$ and on circles of radius $r_{\varepsilon}$, respectively, defined by

$$
\tilde{\mu}_{\varepsilon}^{r_{\varepsilon}}:=\sum_{i=1}^{M_{\varepsilon}} \frac{\chi_{B_{r_{\varepsilon}}\left(x_{i, \varepsilon}\right)}}{\pi r_{\varepsilon}^{2}} \xi_{i, \varepsilon}, \quad \hat{\mu}_{\varepsilon}^{r_{\varepsilon}}:=\sum_{i=1}^{M_{\varepsilon}} \frac{\mathcal{H}^{1}\left\llcorner\partial B_{r_{\varepsilon}}\left(x_{i, \varepsilon}\right)\right.}{2 \pi r_{\varepsilon}} \xi_{i, \varepsilon}
$$

For every $x_{\varepsilon, i}$ in the support set of $\mu_{\varepsilon}$ we define the functions $\tilde{K}_{\varepsilon, i}^{\xi_{\varepsilon, i}}, \hat{K}_{\varepsilon, i}^{\xi_{\varepsilon, i}}: B_{r_{\varepsilon}}\left(x_{\varepsilon, i}\right)$ $\rightarrow \mathbb{M}^{2 \times 2}$ as follows:

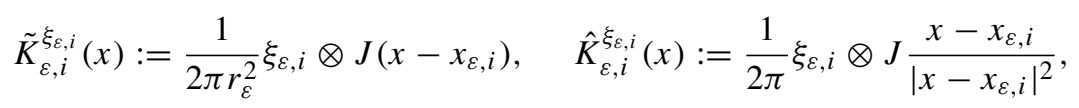

where $J$ is the clockwise rotation of $90^{\circ}$. Finally, we introduce the functions $\tilde{K}_{\varepsilon}^{\mu_{\varepsilon}}, \hat{K}_{\varepsilon}^{\mu_{\varepsilon}}$ : $\Omega \rightarrow \mathbb{R}^{2}$, defined by

$$
\tilde{K}_{\varepsilon}^{\mu_{\varepsilon}}:=\sum_{i=1}^{M_{\varepsilon}} \tilde{K}_{\varepsilon, i}^{\xi_{\varepsilon, i}} \chi_{B_{r_{\varepsilon}}\left(x_{\varepsilon, i}\right)}, \quad \hat{K}_{\varepsilon}^{\mu_{\varepsilon}}:=\sum_{i=1}^{M_{\varepsilon}} \hat{K}_{\varepsilon, i}^{\xi_{\varepsilon, i}} \chi_{B_{r_{\varepsilon}}\left(x_{\varepsilon, i}\right)}
$$

Note that

$$
\operatorname{Curl} \tilde{K}_{\varepsilon}^{\mu_{\varepsilon}}=\tilde{\mu}_{\varepsilon}^{r_{\varepsilon}}-\hat{\mu}_{\varepsilon}^{r_{\varepsilon}}, \quad \operatorname{Curl} \hat{K}_{\varepsilon}^{\mu_{\varepsilon}}=\mu_{\varepsilon}-\hat{\mu}_{\varepsilon}^{r_{\varepsilon}}
$$

Lemma 14. Let $N_{\varepsilon} \rightarrow \infty$ satisfy (14), $\xi:=\sum_{k=1}^{M} \lambda_{k} \xi_{k}$ with $\xi_{k} \in \mathbb{S}, \lambda_{k} \geq 0, \Lambda:=$ $\sum_{k} \lambda_{k}, \mu:=\xi d x$ and $r_{\varepsilon}:=1 /\left(2 \sqrt{\Lambda N_{\varepsilon}}\right)$. Then there exists a sequence of measures $\mu_{\varepsilon}=\sum_{k=1}^{M} \xi_{k} \mu_{\varepsilon}^{k}$ in $X_{\varepsilon}$ with $\mu_{\varepsilon}^{k}$ of the type $\sum_{l=1}^{M_{\varepsilon}^{k}} \delta_{x_{\varepsilon, l}}$ such that $B_{r_{\varepsilon}}(x) \subset \Omega,|x-y| \geq$ $2 r_{\varepsilon}$ for every $x, y$ in the support set of $\mu_{\varepsilon}$, and

$$
\begin{gathered}
\left|\mu_{\varepsilon}^{k}\right| / N_{\varepsilon} \stackrel{*}{\rightarrow} \lambda_{k} d x \quad \text { in } \mathcal{M}(\Omega), \\
\mu_{\varepsilon} / N_{\varepsilon} \stackrel{*}{\rightarrow} \mu \text { in } \mathcal{M}\left(\Omega ; \mathbb{R}^{2}\right), \quad \hat{\mu}_{\varepsilon}^{r_{\varepsilon}} / N_{\varepsilon} \stackrel{*}{\rightarrow} \mu \text { in } \mathcal{M}\left(\Omega ; \mathbb{R}^{2}\right), \\
\tilde{\mu}_{\varepsilon}^{r_{\varepsilon}} / N_{\varepsilon} \stackrel{*}{\rightarrow} \xi \quad \text { in } L^{\infty}\left(\Omega ; \mathbb{R}^{2}\right), \\
\tilde{\mu}_{\varepsilon}^{r_{\varepsilon}} / N_{\varepsilon} \rightarrow \mu, \quad \tilde{\mu}_{\varepsilon}^{r_{\varepsilon}}-\hat{\mu}_{\varepsilon}^{r_{\varepsilon}} / N_{\varepsilon} \rightarrow 0 \quad \text { strongly in } H^{-1}\left(\Omega ; \mathbb{R}^{2}\right) .
\end{gathered}
$$

Proof. First, we prove the assertion for $M=1$ and $\mu=\xi d x$ with $\xi \in \mathbb{S}$.

For $\mu:=\xi d x$ we cover $\mathbb{R}^{2}$ with cubes of size $2 r_{\varepsilon}$, we plug a mass with weight $\xi$ in the center of all of such cubes which are contained in $\Omega$, and we let $\mu_{\varepsilon}$ be the measure obtained through this procedure. Let us prove (71), all the other properties following easily by the definition of $\mu_{\varepsilon}$. Since $\mu-\tilde{\mu}_{\varepsilon}^{r_{\varepsilon}} / N_{\varepsilon}$ converges weakly to zero in $L^{2}\left(\Omega ; \mathbb{R}^{2}\right)$, by the compact embedding of $L^{2}$ in $H^{-1}$ we see that $\mu-\tilde{\mu}_{\varepsilon}^{r_{\varepsilon}} / N_{\varepsilon} \rightarrow 0$ in $H^{-1}\left(\Omega ; \mathbb{R}^{2}\right)$. The fact that $\left(\tilde{\mu}_{\varepsilon}^{r_{\varepsilon}}-\hat{\mu}_{\varepsilon}^{r_{\varepsilon}}\right) / N_{\varepsilon} \rightarrow 0$ strongly in $H^{-1}\left(\Omega ; \mathbb{R}^{2}\right)$ follows directly from 68, 
since $\tilde{K}_{\varepsilon}^{\mu_{\varepsilon}} / N_{\varepsilon} \rightarrow 0$ strongly in $L^{2}\left(\Omega ; \mathbb{R}^{2}\right.$ ) (which can be checked by a very simple estimate).

The general case with $\xi \in \mathbb{R}^{2}$ and $M>1$ will follow by approximating $\mu=\xi d x$ with periodic locally constant measures with weight $\xi_{k}$ on sets with volume fraction $\lambda_{k} / \Lambda$. In each region where the approximating measure is constant we apply the construction above and then we take a diagonal sequence.

We are in a position to prove the $\Gamma$-limsup inequality of Theorem 12 . We will proceed in several steps.

Step 1. The case $\mu \equiv \xi d x$.

Given $\xi \in \mathbb{R}^{2}$ and $\beta \in L^{2}\left(\Omega ; \mathbb{M}^{2 \times 2}\right)$ with $\operatorname{Curl} \beta=\xi d x$, we will construct a recovery sequence $\left\{\mu_{\varepsilon}\right\} \subset X_{\varepsilon}, \beta_{\varepsilon} \in \mathcal{A} \mathcal{S}_{\varepsilon}\left(\mu_{\varepsilon}\right)$, such that $\left(\mu_{\varepsilon}, \beta_{\varepsilon}\right)$ converges to $(\xi d x, \beta)$ in the sense of (50) and (51),

$$
\limsup _{\varepsilon \rightarrow 0} \frac{1}{|\log \varepsilon|^{2}} \int_{\Omega} W\left(\beta_{\varepsilon}\right) d x \leq \int_{\Omega}(W(\beta)+\varphi(\xi)) d x,
$$

and additionally $\left(\beta_{\varepsilon} /|\log \varepsilon|-\beta\right) \cdot t$ tends to zero strongly in $H^{-1 / 2}(\partial \Omega)$.

Let $\varphi$ be the self-energy density defined in (42), and let $\lambda_{k} \geq 0, \xi_{k} \in \mathbb{S}$ be such that $\xi=\sum_{k} \lambda_{k} \xi_{k}$ and

$$
\varphi(\xi)=\sum_{k=1}^{M} \lambda_{k} \psi\left(\xi_{k}\right)
$$

Consider the sequence $\mu_{\varepsilon}:=\sum_{i=1}^{M_{\varepsilon}} \xi_{\varepsilon, i} \delta_{x_{\varepsilon, i}}$ given by Lemma 14 with $N_{\varepsilon}=|\log \varepsilon|$. Note that since $N_{\varepsilon} \rho_{\varepsilon}^{2} \rightarrow 0$, we have $r_{\varepsilon} \gg \rho_{\varepsilon}$, and so $\mu_{\varepsilon} \in X_{\varepsilon}$.

Since the function $\hat{K}_{\varepsilon, i}^{\xi_{\varepsilon, i}}$ defined in 66 belongs to $\mathcal{A S}_{\varepsilon, \rho_{\varepsilon}}\left(\xi_{\varepsilon, i}\right)$ and satisfies condition 40p, by Proposition 8 for every $x_{\varepsilon, i}$ in the support set of $\mu_{\varepsilon}$ we can find a strain $\hat{\beta}_{\varepsilon, i}$ : $\Omega \rightarrow \mathbb{M}^{2 \times 2}$ such that

1) $\hat{\beta}_{\varepsilon, i} \in \mathcal{A S}_{\varepsilon, \rho_{\varepsilon}}\left(\xi_{\varepsilon, i}\right)$,

2) $\hat{\beta}_{\varepsilon, i} \cdot t=\hat{K}_{\varepsilon, i}^{\xi_{\varepsilon, i}} \cdot t$ on $\partial B_{\varepsilon}\left(x_{\varepsilon, i}\right) \cup \partial B_{\rho_{\varepsilon}}\left(x_{\varepsilon, i}\right)$,

3) $|\log \varepsilon|^{-1} \int_{B_{\rho_{\varepsilon}}\left(x_{\varepsilon, i}\right) \backslash B_{\varepsilon}\left(x_{\varepsilon, i}\right)} W\left(\hat{\beta}_{\varepsilon, i}\right) d x=\psi\left(\xi_{\varepsilon, i}\right)(1+o(\varepsilon))$ where $o(\varepsilon) \rightarrow 0$ as $\varepsilon \rightarrow 0$.

Extend $\hat{\beta}_{\varepsilon, i}$ to be $\hat{K}_{\varepsilon, i}^{\xi_{\varepsilon, i}}$ in $B_{r_{\varepsilon}}\left(x_{\varepsilon, i}\right) \backslash B_{\rho_{\varepsilon}}\left(x_{\varepsilon, i}\right)$ and zero in $\Omega \backslash\left(B_{r_{\varepsilon}}\left(x_{\varepsilon, i}\right) \backslash B_{\varepsilon}\left(x_{\varepsilon, i}\right)\right)$, and set

$$
\hat{\beta}_{\varepsilon}:=\sum_{i=1}^{M_{\varepsilon}} \hat{\beta}_{\varepsilon, i} .
$$

Then

4) $\operatorname{Curl} \hat{\beta}_{\varepsilon}=-\hat{\mu}_{\varepsilon}^{r_{\varepsilon}}+\hat{\mu}_{\varepsilon}^{\varepsilon}$, 
where $\hat{\mu}_{\varepsilon}^{r_{\varepsilon}}$ and $\hat{\mu}_{\varepsilon}^{\varepsilon}$ are defined in 65 . Finally, set

$$
\bar{\beta}_{\varepsilon}:=|\log \varepsilon| \beta-\tilde{K}_{\varepsilon}^{\mu_{\varepsilon}}+\hat{\beta}_{\varepsilon},
$$

where $\tilde{K}_{\varepsilon}^{\mu_{\varepsilon}}$ is defined according to 67]. By Lemma 14 and 687, we easily deduce

$$
\begin{aligned}
\operatorname{Curl} \frac{\bar{\beta}_{\varepsilon}}{|\log \varepsilon|}\left\llcorner\Omega_{\varepsilon}\left(\mu_{\varepsilon}\right)\right. & =\mu-\frac{\tilde{\mu}_{\varepsilon}^{r_{\varepsilon}}}{|\log \varepsilon|}+\frac{\hat{\mu}_{\varepsilon}^{r_{\varepsilon}}}{|\log \varepsilon|}-\frac{\hat{\mu}_{\varepsilon}^{r_{\varepsilon}}}{|\log \varepsilon|} \\
& =\mu-\frac{\tilde{\mu}_{\varepsilon}^{r_{\varepsilon}}}{|\log \varepsilon|} \rightarrow 0 \quad \text { in } H^{-1}\left(\Omega ; \mathbb{R}^{2}\right) .
\end{aligned}
$$

Therefore, we can add to $\bar{\beta}_{\varepsilon}$ a vanishing sequence $R_{\varepsilon} /|\log \varepsilon| \rightarrow 0$ in $L^{2}\left(\Omega ; \mathbb{M}^{2 \times 2}\right)$, obtaining the admissible strain

$$
\beta_{\varepsilon}:=\left(\bar{\beta}_{\varepsilon}+R_{\varepsilon}\right) \chi_{\Omega_{\varepsilon}\left(\mu_{\varepsilon}\right)} .
$$

In order to prove that the pair $\left(\mu_{\varepsilon}, \beta_{\varepsilon}\right)$ is the desired recovery sequence we have to check the following properties:

(i) $\beta_{\varepsilon}$ converge to $\beta$ in the sense of definition (51);

(ii) the pair $\left(\beta_{\varepsilon}, \mu_{\varepsilon}\right)$ is a recovery sequence, i.e.,

$$
\lim _{\varepsilon \rightarrow 0} \int_{\Omega} W\left(\beta_{\varepsilon}\right) d x=\int_{\Omega}(W(\beta)+\varphi(\xi)) d x .
$$

To prove (i), we first note that since $M_{\varepsilon} \sim|\log \varepsilon|, r_{\varepsilon} \sim 1 / \sqrt{\varepsilon}$, we have

$$
\int_{\Omega_{\rho_{\varepsilon}}\left(\mu_{\varepsilon}\right)} \frac{\left|\hat{\beta}_{\varepsilon}\right|^{2}}{|\log \varepsilon|} d x=\int_{\Omega_{r_{\varepsilon}}\left(\mu_{\varepsilon}\right) \backslash \Omega_{\rho_{\varepsilon}}\left(\mu_{\varepsilon}\right)} \frac{\left|\hat{K}_{\varepsilon}^{\mu_{\varepsilon}}\right|^{2}}{|\log \varepsilon|} d x \rightarrow 0,
$$

which implies that $\hat{\beta}_{\varepsilon} /|\log \varepsilon|$ is concentrated on the hard core region. Then by Lemma 14 . $\left|\mu_{\varepsilon}^{k}\right| / N_{\varepsilon} \stackrel{*}{\rightarrow} \lambda_{k} d x$ for every $k$, and by property 3 ) we have

$$
\begin{aligned}
\lim _{\varepsilon \rightarrow 0} \frac{1}{|\log \varepsilon|^{2}} \int_{\Omega} W\left(\hat{\beta}_{\varepsilon}\right) d x & =\lim _{\varepsilon \rightarrow 0} \frac{1}{|\log \varepsilon|^{2}} \int_{\Omega \backslash \Omega_{\rho_{\varepsilon}}\left(\mu_{\varepsilon}\right)} W\left(\hat{\beta}_{\varepsilon}\right) d x \\
& =\lim _{\varepsilon \rightarrow 0} \frac{1}{|\log \varepsilon|} \sum_{k=1}^{M}\left|\mu_{\varepsilon}^{k}\right|(\Omega) \psi\left(\xi_{k}\right)=\sum_{k=1}^{M} \lambda_{k} \psi\left(\xi_{k}\right)=\varphi(\xi) .
\end{aligned}
$$

In particular, we deduce that $\hat{\beta}_{\varepsilon} /|\log \varepsilon|$ is bounded in $L^{2}\left(\Omega ; \mathbb{M}^{2 \times 2}\right)$. Since the $L^{2}$ norm of $\hat{\beta}_{\varepsilon} /|\log \varepsilon|$ is concentrated in the hard core region, we conclude that $\hat{\beta}_{\varepsilon} /|\log \varepsilon|$ converges weakly to zero in $L^{2}\left(\Omega ; \mathbb{M}^{2 \times 2}\right)$. On the other hand, one can check directly that $\tilde{K}_{\varepsilon}^{\mu_{\varepsilon}} /|\log \varepsilon|$ converges strongly to zero in $L^{2}(\Omega)$. Recalling that also $R_{\varepsilon} /|\log \varepsilon| \rightarrow 0$, by (75) and (76) we conclude that (i) holds. 
Next we prove (ii), i.e. the pair $\left(\beta_{\varepsilon}, \mu_{\varepsilon}\right)$ is optimal in energy. We have

$$
\lim _{\varepsilon \rightarrow 0} \frac{1}{|\log \varepsilon|^{2}} \int_{\Omega} W\left(\beta_{\varepsilon}\right) d x=\lim _{\varepsilon \rightarrow 0} \frac{1}{|\log \varepsilon|^{2}} \int_{\Omega} W\left(|\log \varepsilon| \beta+\hat{\beta}_{\varepsilon}\right) d x .
$$

Since $\hat{\beta}_{\varepsilon} /|\log \varepsilon| \rightarrow 0$ in $L^{2}\left(\Omega ; \mathbb{M}^{2 \times 2}\right)$, taking into account also 77, , we conclude

$$
\begin{aligned}
\lim _{\varepsilon \rightarrow 0} \frac{1}{|\log \varepsilon|^{2}} \int_{\Omega} W\left(\beta_{\varepsilon}\right) d x & =\lim _{\varepsilon \rightarrow 0} \frac{1}{|\log \varepsilon|^{2}}\left(\int_{\Omega} W(|\log \varepsilon| \beta) d x+\int_{\Omega} W\left(\hat{\beta}_{\varepsilon}\right) d x\right) \\
& =\int_{\Omega}(W(\beta)+\varphi(\xi)) d x .
\end{aligned}
$$

Finally, by the Lipschitz continuity of $\partial \Omega$, from $(75)$ and $(76)$ we also deduce that $\left(\beta_{\varepsilon} /|\log \varepsilon|-\beta\right) \cdot t$ tends to zero strongly in $H^{-1 / 2}(\partial \Omega)$.

Step 2. The case $\mu:=\sum_{l=1}^{L} \chi_{A_{l}} \xi_{l} d x$.

In this step we prove the $\Gamma$-limsup inequality in the case of $\mu$ locally constant, i.e., of the type

$$
\mu:=\sum_{l=1}^{L} \chi_{A_{l}} \xi_{l} d x
$$

where $A_{l}$ are open subsets of $\Omega$ with Lipschitz continuous boundary and $\xi_{i} \in \mathbb{M}^{2 \times 2}$. The construction of the recovery sequence is based on classical localization arguments in $\Gamma$-convergence and takes advantage of the previous step.

Set $\beta_{l}:=\beta\left\llcorner A_{l}\right.$, and let $\mu_{l, \varepsilon}, \beta_{l, \varepsilon}$ be the recovery sequence given by Step 1 applied with $\Omega$ replaced by $A_{l}$, with $\beta=\beta_{l}, \mu=\xi_{l} d x$. Finally define $\bar{\beta}_{\varepsilon}: \Omega \rightarrow \mathbb{M}^{2 \times 2}$ and $\mu_{\varepsilon} \in \mathcal{M}\left(\Omega ; \mathbb{R}^{2}\right)$ as follows:

$$
\bar{\beta}_{\varepsilon}(x):=\beta_{l, \varepsilon} \quad \text { if } x \in A_{l}, \quad \mu_{\varepsilon}:=\sum_{l} \mu_{l, \varepsilon} .
$$

By construction we have $\mu_{\varepsilon} \in X_{\varepsilon}$. Moreover since

$$
\frac{1}{|\log \varepsilon|} \| \operatorname{Curl} \bar{\beta}_{\varepsilon}\left\llcorner\Omega_{\varepsilon}\left(\mu_{\varepsilon}\right)\left\|_{H^{-1}\left(\Omega ; \mathbb{R}^{2}\right)} \leq \sum_{l}\right\| \frac{\beta_{\varepsilon, l}}{|\log \varepsilon|}-\beta \|_{H^{-1 / 2}\left(\partial A_{l}\right)},\right.
$$

from the previous step it easily follows that

$$
\frac{1}{|\log \varepsilon|} \operatorname{Curl} \bar{\beta}_{\varepsilon}\left\llcorner\Omega_{\varepsilon}\left(\mu_{\varepsilon}\right) \rightarrow 0\right.
$$

strongly in $H^{-1}\left(\Omega ; \mathbb{R}^{2}\right)$. Therefore we can easily modify this sequence $\bar{\beta}_{\varepsilon}$ by adding a vanishing perturbation in order to obtain the desired recovery sequence $\beta_{\varepsilon}$.

Step 3. The general case.

In this step we show how to construct a recovery sequence in the general case (i.e., for a general dislocation measure $\mu \in \mathcal{M}\left(\Omega ; \mathbb{R}^{2}\right)$ ). 
Let $(\mu, \beta)$ be given in the domain of the $\Gamma$-limit $\mathcal{F}$. In view of the previous step and by standard density arguments in $\Gamma$-convergence, it is enough to construct sequences $\left(\mu_{n}, \beta_{n}\right)$ with $\operatorname{Curl} \beta_{n}=\mu_{n}$ and with $\mu_{n}$ locally constant as in 78 such that

$$
\beta_{n} \rightarrow \beta \text { in } L^{2}\left(\Omega ; \mathbb{M}^{2 \times 2}\right), \quad \mu_{n} \stackrel{*}{\rightarrow} \mu \text { in } \mathcal{M}\left(\Omega ; \mathbb{M}^{2 \times 2}\right) \quad \text { and } \quad\left|\mu_{n}\right|(\Omega) \rightarrow|\mu|(\Omega) .
$$

Indeed, under these convergence assumptions we get the convergence of the corresponding energies, i.e.,

$$
\lim _{n \rightarrow \infty} \mathcal{F}\left(\beta_{n}, \mu_{n}\right)=\mathcal{F}(\beta, \mu) .
$$

By standard reflection arguments we can extend the strain $\beta$ to a function $\beta_{A}$ defined in a neighborhood $A$ of $\Omega$, such that $\operatorname{Curl} \beta_{A}=\mu_{A}$ is a measure on $A$ and moreover $\left|\mu_{A}\right|(\partial \Omega)=0$.

Let $\rho_{h}$ be a sequence of mollifiers, and define

$$
f_{h}:=\beta_{A} * \rho_{h}\left\llcorner\Omega, \quad g_{h}:=\mu_{A} * \rho_{h}\llcorner\Omega .\right.
$$

For $h$ large enough these objects are well defined in $\Omega$ and Curl $f_{h}=g_{h}$. Clearly

$$
f_{h} \rightarrow \beta \text { in } L^{2}\left(\Omega ; \mathbb{M}^{2 \times 2}\right), \quad g_{h} d x \stackrel{*}{\rightarrow} \mu \text { in } \mathcal{M}\left(\Omega ; \mathbb{R}^{2}\right) .
$$

Moreover, since $\left|\mu_{A}\right|(\partial \Omega)=0$, we have

$$
\left|g_{h} d x\right|(\Omega) \rightarrow|\mu|(\Omega) .
$$

Next, we approximate every $g_{h}$ by locally constant functions; more precisely, we consider a locally constant function $g_{h, k}$ such that

$$
\left|g_{h, k}-g_{h}\right|_{L^{\infty}\left(\Omega ; \mathbb{R}^{2}\right)} \rightarrow 0 \quad \text { as } k \rightarrow \infty, \quad \text { and } \quad \int_{\Omega}\left(g_{h, k}-g_{h}\right) d x=0 .
$$

Let $r_{h, k}$ be the solution of the following problem:

$$
\begin{cases}\operatorname{Curl} r_{h, k}=g_{h, k}-g_{h} & \text { in } \Omega, \\ \operatorname{Div} r_{h, k}=0 & \text { in } \Omega, \\ r_{h, k} \cdot t=0 & \text { in } \partial \Omega .\end{cases}
$$

By standard elliptic estimates we have

$$
\left|r_{h, k}\right|_{L^{2}\left(\Omega ; \mathbb{M}^{2 \times 2}\right)} \leq C\left|g_{h, k}-g_{h}\right|_{L^{2}\left(\Omega ; \mathbb{R}^{2}\right)} .
$$

Finally, we set $f_{h, k}:=f_{h}+r_{h, k}$. By (84) we have Curl $f_{h, k}=g_{h, k}$. Moreover, by (83), 85. we have

$$
f_{h, k} \rightarrow f_{h} \quad \text { in } L^{2}\left(\Omega ; \mathbb{M}^{2 \times 2}\right) \text { as } k \rightarrow \infty .
$$

By (81), 83), 86, using a diagonal argument we can find a sequence $\left(\mu_{n}, \beta_{n}\right)$ satisfying (79), and therefore (80). 


\section{The subcritical case $\left(N_{\varepsilon} \ll|\log \varepsilon|\right)$}

In this section we study the asymptotic behavior of the energy functionals $E_{\varepsilon}$ defined in (19) in the case of dilute dislocations, i.e., for $N_{\varepsilon} \ll|\log \varepsilon|$. In terms of $\Gamma$-convergence, it means that we rescale $E_{\varepsilon}$ with a prefactor $N_{\varepsilon}|\log \varepsilon|$, with $N_{\varepsilon} \ll|\log \varepsilon|$. As discussed in Section 2, in this case the self-energy for minimizing sequences is predominant with respect to the interaction energy (see Remark 16).

In contrast to the critical case, the prefactors of strains and dislocation measures in the subcritical case are different. Indeed, the natural rescaling for the dislocation measures is given by $N_{\varepsilon}$. On the other hand, in order to observe the effect of the diffuse energy associated with a sequence $\left(\mu_{\varepsilon}, \beta_{\varepsilon}\right)$ with bounded energy we have to rescale the strains by $\left(N_{\varepsilon}|\log \varepsilon|\right)^{1 / 2}$. These two quantities clearly coincide only in the critical case $N_{\varepsilon} \equiv$ $|\log \varepsilon|$. The effect of a different rescaling for strains and dislocation measures is that, in the limit configuration, $\mu$ and $\beta$ are independent variables, i.e., the compatibility condition $\operatorname{Curl} \beta=\mu$ disappears in this limit. Actually the admissible strains in the limit are always gradients, i.e., $\operatorname{Curl} \beta=0$. Heuristically this is a consequence of the fact that the total variation of $\operatorname{Curl} \beta_{\varepsilon}$ is of order $N_{\varepsilon}$, so that $\operatorname{Curl}\left(\beta_{\varepsilon} /\left(N_{\varepsilon}|\log \varepsilon|\right)^{1 / 2}\right)$ vanishes.

The candidate $\Gamma$-limit of the functionals $\mathcal{F}_{\varepsilon}^{\text {dilute }}: \mathcal{M}\left(\Omega ; \mathbb{R}^{2}\right) \times L^{2}\left(\Omega ; \mathbb{M}^{2 \times 2}\right)$ defined in 21] is the functional $\mathcal{F}^{\text {dilute }}$ defined by

$$
\mathcal{F}^{\text {dilute }}(\mu, \beta):= \begin{cases}\int_{\Omega} W(\beta) d x+\int_{\Omega} \varphi\left(\frac{d \mu}{d|\mu|}\right) d|\mu| & \text { if } \operatorname{Curl} \beta=0 \\ +\infty & \text { otherwise in } L^{2}\left(\Omega ; \mathbb{M}^{2 \times 2}\right) .\end{cases}
$$

The precise $\Gamma$-convergence result is the following.

Theorem 15. Let $N_{\varepsilon} \rightarrow \infty$ be such that $N_{\varepsilon} /|\log \varepsilon| \rightarrow 0$. Then the following $\Gamma$-convergence result holds.

(i) (Compactness) Let $\varepsilon_{n} \rightarrow 0$ and let $\left\{\left(\mu_{n}, \beta_{n}\right)\right\}$ be a sequence in $\mathcal{M}\left(\Omega ; \mathbb{R}^{2}\right) \times$ $L^{2}\left(\Omega ; \mathbb{M}^{2 \times 2}\right)$ such that $\mathcal{F}_{\varepsilon_{n}}^{\text {dilute }}\left(\mu_{n}, \beta_{n}\right) \leq E$ for some positive constant $E$ independent of $n$. Then there exist $\mu \in \mathcal{M}\left(\Omega ; \mathbb{R}^{2}\right)$ and $\beta \in L^{2}\left(\Omega ; \mathbb{M}^{2 \times 2}\right)$, with $\operatorname{Curl} \beta=0$, such that (up to a subsequence)

$$
\begin{gathered}
\frac{1}{N_{\varepsilon_{n}}} \mu_{n} \stackrel{*}{\rightarrow} \mu \quad \text { in } \mathcal{M}\left(\Omega ; \mathbb{R}^{2}\right), \\
\frac{1}{\left(N_{\varepsilon_{n}}\left|\log \varepsilon_{n}\right|\right)^{1 / 2}} \beta_{n} \rightarrow \beta \quad \text { in } L^{2}\left(\Omega ; \mathbb{M}^{2 \times 2}\right) .
\end{gathered}
$$

(ii) ( $\Gamma$-convergence) The functionals $\mathcal{F}_{\varepsilon} \Gamma$-converge as $\varepsilon \rightarrow 0$, with respect to the convergence in (88) and (89), to the functional $\mathcal{F}^{\text {dilute }}$ defined in 87). More precisely, the following inequalities hold:

- ( $\Gamma$-liminf inequality) for every $(\mu, \beta) \in \mathcal{M}\left(\Omega ; \mathbb{R}^{2}\right) \times L^{2}\left(\Omega ; \mathbb{M}^{2 \times 2}\right)$ with Curl $\beta$ $=0$ and for every sequence $\left(\mu_{\varepsilon}, \beta_{\varepsilon}\right) \in X_{\varepsilon} \times L^{2}\left(\Omega ; \mathbb{M}^{2 \times 2}\right)$ satisfying , 88 and [89, we have

$$
\liminf _{\varepsilon \rightarrow 0} \mathcal{F}_{\varepsilon}^{\text {dilute }}\left(\mu_{\varepsilon}, \beta_{\varepsilon}\right) \geq \mathcal{F}^{\text {dilute }}(\mu, \beta)
$$


- $\left(\Gamma\right.$-limsup inequality) given $(\mu, \beta) \in \mathcal{M}\left(\Omega ; \mathbb{R}^{2}\right) \times L^{2}\left(\Omega ; \mathbb{M}^{2 \times 2}\right)$ with Curl $\beta=0$, there exists $\left(\mu_{\varepsilon}, \beta_{\varepsilon}\right) \in X_{\varepsilon} \times L^{2}\left(\Omega ; \mathbb{M}^{2 \times 2}\right)$ satisfying (88) and (89) such that

$$
\limsup _{\varepsilon \rightarrow 0} \mathcal{F}_{\varepsilon}^{\text {dilute }}\left(\mu_{\varepsilon}, \beta_{\varepsilon}\right) \leq \mathcal{F}^{\text {dilute }}(\mu, \beta) .
$$

Remark 16. The independence of strains and dislocation measures in the $\Gamma$-limit is a consequence of the fact that, in the dilute regime, the interaction energy is a term of lower order than the self-energy. Indeed, by the $\Gamma$-convergence result stated in Theorem 15 we immediately deduce that the functionals $\mathcal{E}_{\varepsilon}^{\text {dilute }}$ defined by

$$
\mathcal{E}_{\varepsilon}^{\text {dilute }}(\mu):=\min _{\beta \in \mathcal{A \mathcal { S }}_{\varepsilon}(\Omega)} \mathcal{F}_{\varepsilon}^{\text {dilute }}(\mu, \beta)
$$

$\Gamma$-converge (as $\varepsilon \rightarrow 0$ ) to the functional $\mathcal{E}^{\text {dilute }}: \mathcal{M}\left(\Omega ; \mathbb{R}^{2}\right) \rightarrow \mathbb{R}$ defined by

$$
\mathcal{E}^{\text {dilute }}(\mu):=\int_{\Omega} \varphi\left(\frac{d \mu}{d|\mu|}\right) d|\mu| \text {. }
$$

The energy $\mathcal{E}^{\text {dilute }}(\mu)$ represents the energy stored in the crystal induced by the distribution $\mu$ of dislocations in the dilute regime, and it is given only by the self-energy.

The proof of Theorem 15 follows the lines of the proof of Theorem 12 . For the reader's convenience we sketch its main steps.

Proof of Theorem 15 The compactness property of rescaled strains and dislocation measures stated in 88$]$ and $(89)$ can be proved with minor changes as in the critical case. Let us prove that in this case $\operatorname{Curl} \beta=0$. Let $\varphi \in C_{0}^{1}(\Omega)$ and let $\left\{\varphi_{n}\right\} \subset H_{0}^{1}(\Omega)$ be a sequence converging to $\varphi$ uniformly and strongly in $H_{0}^{1}(\Omega)$ and satisfying the property

$$
\varphi_{n} \equiv \varphi\left(x_{i, n}\right) \quad \text { in } B_{\varepsilon_{n}}\left(x_{i, n}\right) \text { for every } x_{i, n} \text { in the support set of } \mu_{n} .
$$

By Remark 1 we have

$$
\begin{aligned}
\langle\operatorname{Curl} \beta, \varphi\rangle= & \lim _{\varepsilon_{n} \rightarrow 0} \frac{1}{N_{\varepsilon_{n}}^{1 / 2}\left|\log \varepsilon_{n}\right|^{1 / 2}}\left\langle\operatorname{Curl} \beta_{n}, \varphi_{n}\right\rangle \\
& =\lim _{\varepsilon_{n} \rightarrow 0} \frac{N_{\varepsilon_{n}}^{1 / 2}}{\left|\log \varepsilon_{n}\right|^{1 / 2}} \frac{1}{N_{\varepsilon_{n}}} \int_{\Omega} \varphi_{n} d \mu_{n}=\lim _{\varepsilon_{n} \rightarrow 0} \frac{N_{\varepsilon_{n}}^{1 / 2}}{\left|\log \varepsilon_{n}\right|^{1 / 2}} \int_{\Omega} \varphi d \mu=0,
\end{aligned}
$$

from which we deduce $\operatorname{Curl} \beta=0$.

Concerning the $\Gamma$-convergence result, the proof of the $\Gamma$-liminf inequality is identical to that of the critical case, so that we pass directly to the proof of the $\Gamma$-limsup inequality.

As in the critical case, classical localization arguments reduce the problem to the case of $\mu$ constant. (Note that the density argument used in the critical case is even easier in the subcritical case, since no admissibility condition $\operatorname{Curl} \beta=\mu$ is required.) 
The proof of the $\Gamma$-limsup inequality reduces to finding a sequence $\left\{\mu_{\varepsilon}\right\} \subset$ $X_{\varepsilon}$, with $\left(1 / N_{\varepsilon}\right) \mu_{\varepsilon} \stackrel{*}{\rightarrow} \xi d x$ in $\mathcal{M}\left(\Omega ; \mathbb{R}^{2}\right)$, and a sequence $\beta_{\varepsilon} \in \mathcal{A} \mathcal{S}_{\varepsilon}\left(\mu_{\varepsilon}\right)$, with $\left(1 /\left(N_{\varepsilon}|\log \varepsilon|\right)^{1 / 2}\right) \beta_{\varepsilon} \rightarrow \beta$ in $L^{2}\left(\Omega ; \mathbb{M}^{2 \times 2}\right)$, such that

$$
\limsup _{\varepsilon \rightarrow 0} \frac{1}{N_{\varepsilon}|\log \varepsilon|} \int_{\Omega} W\left(\beta_{\varepsilon}\right) d x \leq \int_{\Omega}(W(\beta)+\varphi(\xi)) d x,
$$

and satisfying the additional requirement that $\left(\beta_{\varepsilon} /|\log \varepsilon|-\beta\right) \cdot t$ tends to zero strongly in $H^{-1 / 2}(\partial \Omega)$.

Consider the sequence $\mu_{\varepsilon}:=\sum_{i=1}^{M_{\varepsilon}} \xi_{\varepsilon, i} \delta_{x_{\varepsilon, i}}$ given by Lemma 14 . Construct the functions $\hat{\beta}_{\varepsilon}: \Omega \rightarrow \mathbb{R}^{2}$ as in 747. Then set

$$
\bar{\beta}_{\varepsilon}:=\left(N_{\varepsilon}|\log \varepsilon|\right)^{1 / 2} \beta-\tilde{K}_{\varepsilon}^{\mu_{\varepsilon}}+\hat{\beta}_{\varepsilon},
$$

where $\tilde{K}_{\varepsilon}^{\mu_{\varepsilon}}$ is defined according to 67). By 68,

$$
\operatorname{Curl} \bar{\beta}_{\varepsilon}\left\llcorner\Omega_{\varepsilon}\left(\mu_{\varepsilon}\right)=-\tilde{\mu}_{\varepsilon}^{r_{\varepsilon}} .\right.
$$

By its definition the density of the measure $\tilde{\mu}_{\varepsilon}^{r_{\varepsilon}} /\left(N_{\varepsilon}|\log \varepsilon|\right)^{1 / 2}$ tends to zero uniformly as $\varepsilon \rightarrow 0$. Therefore, we can add to $\bar{\beta}_{\varepsilon}$ a sequence $R_{\varepsilon}$ in $L^{2}\left(\Omega ; \mathbb{M}^{2 \times 2}\right)$ with $R_{\varepsilon} /\left(N_{\varepsilon}|\log \varepsilon|\right)^{1 / 2}$ $\rightarrow 0$, obtaining the admissible strain

$$
\beta_{\varepsilon}:=\left(\bar{\beta}_{\varepsilon}+R_{\varepsilon}\right) \chi \Omega_{\varepsilon}\left(\mu_{\varepsilon}\right) .
$$

In order to prove that the pair $\left(\mu_{\varepsilon}, \beta_{\varepsilon}\right)$ is the desired recovery sequence, we have to check the following properties:

(i) $\beta_{\varepsilon}$ converge to $\beta$ in the sense of definition (89);

(ii) the pair $\left(\beta_{\varepsilon}, \mu_{\varepsilon}\right)$ is a recovery sequence, i.e.,

$$
\lim _{\varepsilon \rightarrow 0} \int_{\Omega} W\left(\beta_{\varepsilon}\right) d x=\int_{\Omega}(W(\beta)+\varphi(\xi)) d x .
$$

To prove (i), recalling that by Lemma $14,\left|\mu_{\varepsilon}^{k}\right| / N_{\varepsilon} \stackrel{*}{\rightarrow} \lambda_{k} d x$ for every $k$, we have

$$
\lim _{\varepsilon \rightarrow 0} \frac{1}{N_{\varepsilon}|\log \varepsilon|} \int_{\Omega} W\left(\hat{\beta}_{\varepsilon}\right) d x=\lim _{\varepsilon \rightarrow 0} \frac{1}{N_{\varepsilon}} \sum_{k=1}^{M}\left|\mu_{\varepsilon}^{k}\right|(\Omega) \psi\left(\xi_{k}\right)=\sum_{k=1}^{M} \lambda_{k} \psi\left(\xi_{k}\right)=\varphi(\xi)
$$

We deduce that $\hat{\beta}_{\varepsilon} /\left(N_{\varepsilon}|\log \varepsilon|\right)^{1 / 2}$ is bounded in $L^{2}\left(\Omega ; \mathbb{M}^{2 \times 2}\right)$. As in the critical case, the $L^{2}$ norm of $\hat{\beta}_{\varepsilon} /|\log \varepsilon|$ is concentrated in the hard core region, so that $\hat{\beta}_{\varepsilon} /|\log \varepsilon|$ converges weakly to zero in $L^{2}\left(\Omega ; \mathbb{M}^{2 \times 2}\right)$. On the other hand, one can check directly that $\tilde{K}_{\varepsilon}^{\mu_{\varepsilon}} /\left(N_{\varepsilon}|\log \varepsilon|\right)^{1 / 2}$ converges strongly to zero in $L^{2}(\Omega)$, from which property (i) follows.

Concerning (ii), we prove that the pair $\left(\beta_{\varepsilon}, \mu_{\varepsilon}\right)$ is optimal in energy. We have

$$
\lim _{\varepsilon \rightarrow 0} \frac{1}{N_{\varepsilon}|\log \varepsilon|} \int_{\Omega} W\left(\beta_{\varepsilon}\right) d x=\lim _{\varepsilon \rightarrow 0} \frac{1}{N_{\varepsilon}|\log \varepsilon|} \int_{\Omega} W\left(|\log \varepsilon| \beta+\hat{\beta}_{\varepsilon}\right) d x .
$$


Since $\hat{\beta}_{\varepsilon} /|\log \varepsilon| \rightarrow 0$ in $L^{2}\left(\Omega ; \mathbb{M}^{2 \times 2}\right)$, taking into account also 93 , we conclude

$$
\begin{aligned}
\lim _{\varepsilon \rightarrow 0} \frac{1}{N_{\varepsilon}|\log \varepsilon|} \int_{\Omega} & W\left(\beta_{\varepsilon}\right) d x \\
& =\lim _{\varepsilon \rightarrow 0} \frac{1}{N_{\varepsilon}|\log \varepsilon|}\left(\int_{\Omega} W\left(\left(N_{\varepsilon}|\log \varepsilon|\right)^{1 / 2} \beta\right) d x+\int_{\Omega} W\left(\hat{\beta}_{\varepsilon}\right) d x\right) \\
& =\int_{\Omega}(W(\beta)+\varphi(\xi)) d x .
\end{aligned}
$$

Finally, by the Lipschitz continuity of $\partial \Omega$, from 91 and 92 we also deduce that $\left(\beta_{\varepsilon} /\left(N_{\varepsilon}|\log \varepsilon|\right)^{1 / 2}-\beta\right) \cdot t$ tends to zero strongly in $H^{-1 / 2}(\partial \Omega)$.

Remark 17. The case $N_{\varepsilon} \leq C$ has been considered in [6], where the asymptotic behavior of the elastic energy for a fixed distribution of dislocations $\mu_{\varepsilon} \equiv \mu$ is provided up to the second order, and in [21], where the problem of $\Gamma$-convergence induced by screw dislocations is addressed (with $E_{\varepsilon}(\beta):=\|\beta\|_{2}^{2}$ ), without any assumption involving the notion of hard core region (essentially with $\rho_{\varepsilon} \approx \varepsilon$ ).

We could extend the result given by Theorem 15 to the case $N_{\varepsilon} \leq C$, obtaining a $\Gamma$-limit which still has the form as in (87), but with $\mu:=\sum_{i=1}^{M} \xi_{i} \delta_{x_{i}}$, where $\xi_{i} \in \mathbb{S}$, and with $\varphi: \mathbb{S} \rightarrow \mathbb{R}$ defined now by

$$
\varphi(\xi):=\inf \left\{\sum_{k=1}^{N} \psi\left(\xi_{k}\right): \sum_{k=1}^{N} \xi_{k}=\xi, N \in \mathbb{N}, \xi_{k} \in \mathbb{S}\right\} .
$$

\section{The supercritical case $\left(N_{\varepsilon} \gg|\log \varepsilon|\right)$}

In this section we study the asymptotic behavior of the energy functionals $E_{\varepsilon}$ defined in (19) in the supercritical case, i.e., for $N_{\varepsilon} \gg|\log \varepsilon|$. In terms of $\Gamma$-convergence, it means that we rescale $E_{\varepsilon}$ by $N_{\varepsilon}^{2}$, obtaining the rescaled energy functionals $\mathcal{F}_{\varepsilon}^{\text {super }}$ defined in (23). As discussed in Section 2 in this case the interaction energy for minimizing sequences is predominant with respect to the self-energy.

The natural rescaling for the strains in this case is given by $N_{\varepsilon}$, but we do not have any control on the total variation of the dislocation measure. As a consequence we will get a limit energy $\mathcal{F}^{\text {super }}$ defined on $L^{2}\left(\Omega ; \mathbb{M}_{\text {sym }}^{2 \times 2}\right)$ depending on strains given by

$$
\mathcal{F}^{\text {super }}\left(\beta^{\text {sym }}\right):=\int_{\Omega} W\left(\beta^{\text {sym }}\right) d x \quad \text { if } \beta^{\text {sym }} \in L^{2}\left(\Omega ; \mathbb{M}_{\text {sym }}^{2 \times 2}\right),
$$

where $\mathbb{M}_{\text {sym }}^{2 \times 2}$ denotes the class of symmetric matrices in $\mathbb{M}^{2 \times 2}$. The precise $\Gamma$-convergence result is the following.

Theorem 18. Let $N_{\varepsilon}$ be such that $N_{\varepsilon} /|\log \varepsilon| \rightarrow \infty$ as $\varepsilon \rightarrow 0$. Then the following $\Gamma-$ convergence result holds: 
(i) (Compactness) Let $\varepsilon_{n} \rightarrow 0$ and let $\left\{\left(\mu_{n}, \beta_{n}\right)\right\}$ be a sequence in $\mathcal{M}\left(\Omega ; \mathbb{R}^{2}\right) \times$ $L^{2}\left(\Omega ; \mathbb{M}^{2 \times 2}\right)$ such that $\mathcal{F}_{\varepsilon_{n}}^{\text {super }}\left(\mu_{n}, \beta_{n}\right) \leq E$ for some positive constant $E$ independent of $n$. Then there exists a strain $\beta^{\text {sym }} \in L^{2}\left(\Omega ; \mathbb{M}^{2 \times 2}\right)$ such that (up to a subsequence)

$$
\frac{1}{N_{\varepsilon_{n}}} \beta_{n}^{\mathrm{sym}} \rightarrow \beta^{\mathrm{sym}} \quad \text { in } L^{2}\left(\Omega ; \mathbb{M}_{\mathrm{sym}}^{2 \times 2}\right) .
$$

(ii) ( $\Gamma$-convergence) The functionals $\mathcal{F}_{\varepsilon}^{\text {super }} \Gamma$-converge as $\varepsilon \rightarrow 0$, with respect to the convergence in (95), to the functional $\mathcal{F}^{\text {super }}$ defined in (94). More precisely, the following inequalities hold:

- ( $\Gamma$-liminf inequality) for every $\beta^{\mathrm{sym}} \in L^{2}\left(\Omega ; \mathbb{M}_{\mathrm{sym}}^{2 \times 2}\right)$ and for every sequence $\left(\mu_{\varepsilon}, \beta_{\varepsilon}\right) \in X_{\varepsilon} \times L^{2}\left(\Omega ; \mathbb{M}^{2 \times 2}\right)$ satisfying $(95)$ we have

$$
\liminf _{\varepsilon \rightarrow 0} \mathcal{F}_{\varepsilon}^{\text {super }}\left(\mu_{\varepsilon}, \beta_{\varepsilon}\right) \geq \mathcal{F}^{\text {super }}\left(\beta^{\text {sym }}\right)
$$

- ( $\Gamma$-limsup inequality) given $\beta^{\text {sym }} \in L^{2}\left(\Omega ; \mathbb{M}_{\text {sym }}^{2 \times 2}\right)$ there exists $\left(\mu_{\varepsilon}, \beta_{\varepsilon}\right) \in X_{\varepsilon} \times$ $L^{2}\left(\Omega ; \mathbb{M}^{2 \times 2}\right)$ satisfying 95 such that

$$
\limsup _{\varepsilon \rightarrow 0} \mathcal{F}_{\varepsilon}^{\text {super }}\left(\mu_{\varepsilon}, \beta_{\varepsilon}\right) \leq \mathcal{F}^{\text {super }}\left(\beta^{\text {sym }}\right) .
$$

Proof. The compactness property is simply due to the usual apriori $L^{2}$ bound for the strains $\beta_{\varepsilon_{n}}^{\text {sym }}$, while the $\Gamma$-liminf inequality comes simply by lower semicontinuity.

The main difference with respect to the previous energy regimes is in the proof of the $\Gamma$-limsup inequality. Again the strategy is to approximate a special class of limiting configurations and then to proceed by density, but in this case it will be more convenient to approximate the strains $\beta$ with $C^{1}$ functions, so that their curl's are continuous.

Thus, fix $\beta \in L^{2}\left(\Omega ; \mathbb{M}^{2 \times 2}\right)$ such that $\operatorname{Curl} \beta$ is a measure $\mu$ of the type $\mu=g(x) d x$ with $g$ continuous and let us construct a sequence $\left\{\mu_{\varepsilon}\right\} \subset X_{\varepsilon}(\Omega)$ and a sequence $\beta_{\varepsilon} \in$ $\mathcal{A S}_{\varepsilon}\left(\mu_{\varepsilon}\right)$, with $\beta_{\varepsilon} / N_{\varepsilon} \rightarrow \beta$ in $L^{2}\left(\Omega ; \mathbb{M}^{2 \times 2}\right)$, such that the following $\Gamma$-limsup inequality holds true:

$$
\limsup _{\varepsilon \rightarrow 0} \frac{1}{N_{\varepsilon}^{2}} \int_{\Omega} W\left(\beta_{\varepsilon}\right) d x \leq \int_{\Omega} W(\beta) d x .
$$

Arguing as in the proof of Lemma 14, it is easy to prove that there exist $C \in \mathbb{R}$ depending only on $\|g\|_{L^{\infty}\left(\Omega ; \mathbb{R}^{2}\right)}$ and a sequence of measures $\mu_{\varepsilon}:=\sum_{i=1}^{M_{\varepsilon}} \xi_{i, \varepsilon} \delta_{x_{i, \varepsilon}} \in X_{\varepsilon}$, with $\left|\xi_{i, \varepsilon}\right| \leq C$, such that, setting $r_{\varepsilon}:=C / \sqrt{N_{\varepsilon}}$, we have $B_{r_{\varepsilon}}\left(x_{i, j}\right) \in \Omega,\left|x_{i, \varepsilon}-x_{j, \varepsilon}\right|$ $\geq 2 r_{\varepsilon}$ for every $x_{i, \varepsilon}, x_{j, \varepsilon}$ in the support set of $\mu_{\varepsilon}$, and, finally,

$$
\mu_{\varepsilon} / N_{\varepsilon} \stackrel{*}{\rightarrow} \mu \quad \text { in } \mathcal{M}\left(\Omega ; \mathbb{R}^{2}\right), \quad \hat{\mu}_{\varepsilon}^{r_{\varepsilon}} / N_{\varepsilon} \rightarrow \mu \quad \text { strongly in } H^{-1}\left(\Omega ; \mathbb{R}^{2}\right),
$$

where $\hat{\mu}_{\varepsilon}$ is defined according to 65). Consider the functions $\hat{K}_{\varepsilon}^{\mu_{\varepsilon}}$ defined in (67) and set $\bar{\beta}_{\varepsilon}:=N_{\varepsilon} \beta+\hat{K}_{\varepsilon}^{\mu_{\varepsilon}}$. By 68 we deduce

$$
\operatorname{Curl} \frac{\bar{\beta}_{\varepsilon}}{N_{\varepsilon}}\left\llcorner\Omega_{\varepsilon}\left(\mu_{\varepsilon}\right)=\mu-\frac{\hat{\mu}_{\varepsilon}^{r_{\varepsilon}}}{N_{\varepsilon}} \rightarrow 0 \quad \text { in } H^{-1}\left(\Omega ; \mathbb{R}^{2}\right)\right. \text {. }
$$


Therefore, we can add to $\bar{\beta}_{\varepsilon}$ a vanishing sequence $R_{\varepsilon} / N_{\varepsilon} \rightarrow 0$ in $L^{2}\left(\Omega ; \mathbb{M}^{2 \times 2}\right)$, obtaining the admissible strain

$$
\beta_{\varepsilon}:=\left(\bar{\beta}_{\varepsilon}+R_{\varepsilon}\right) \chi_{\Omega_{\varepsilon}\left(\mu_{\varepsilon}\right)} .
$$

In order to prove that the pair $\left(\mu_{\varepsilon}, \beta_{\varepsilon}\right)$ is the desired recovery sequence it is enough to observe that $\hat{K}_{\varepsilon}^{\mu_{\varepsilon}} / N_{\varepsilon} \rightarrow 0$ in $L^{2}\left(\Omega ; \mathbb{M}^{2 \times 2}\right)$. Indeed, by construction we have $M_{\varepsilon} \leq C N_{\varepsilon}$, and therefore

$$
\lim _{\varepsilon \rightarrow 0} \frac{1}{N_{\varepsilon}^{2}} \int_{\Omega_{\varepsilon}}\left|\hat{K}_{\varepsilon}^{\mu_{\varepsilon}}\right|^{2} d x \leq \lim _{\varepsilon \rightarrow 0} \frac{C}{N_{\varepsilon}^{2}} M_{\varepsilon}|\log \varepsilon| \leq \lim _{\varepsilon \rightarrow 0} C \frac{|\log \varepsilon|}{N_{\varepsilon}}=0 .
$$

Remark 19. Note that in the supercritical regime we cannot have a compactness property for the anti-symmetric part of the admissible strains $\beta_{\varepsilon}$, and indeed it is easy to exhibit examples where $\left\|\beta_{\varepsilon}^{\text {sym }} / N_{\varepsilon}\right\|_{L^{2}\left(\Omega ; \mathbb{M}_{\mathrm{sym}}^{2 \times 2}\right)} \leq C$ and $\left\|\beta_{\varepsilon}^{\text {skew }} / N_{\varepsilon}\right\|_{L^{2}\left(\Omega ; \mathbb{M}^{2 \times 2}\right)} \rightarrow \infty$. Note that, since we do not have any control on the mass of $\operatorname{Curl} \beta_{\varepsilon}$, we cannot apply Theorem 11

Acknowledgments. We want to thank Paolo Cermelli for many fruitful discussions that have been essential in formulating the problem. We also thank Adriano Pisante for stimulating interactions. We thank the Center for Nonlinear Analysis (NSF Grants No. DMS-0405343 and DMS-0635983) for its support during the preparation of this paper. The research of G. Leoni was partially supported by the National Science Foundation under Grants No. DMS-0405423 and DMS-0708039.

\section{References}

[1] Ariza, M. P., Ortiz, M.: Discrete crystal elasticity and discrete dislocations in crystals. Arch. Ration. Mech. Anal. 178, 149-226 (2006) Zbl 1115.74012 MR 2186424

[2] Bacon, D. J., Barnett, D. M., Scattergood, R. O.: Anisotropic continuum theory of lattice defects. Progress in Materials Science 23, 51-262 (1978)

[3] Bourgain, J., Brezis, H.: New estimates for elliptic equations and Hodge type systems. J. Eur. Math. Soc. 9, 277-315 (2007) Zbl pre05177827 MR 2293957

[4] Bethuel, F., Brezis, H., Hélein, F.: Ginzburg-Landau Vortices. Progr. Nonlinear Differential Equations Appl. 13, Birkhäuser Boston, Boston, MA (1994). Zbl 0802.35142 MR 1269538

[5] Brezis, H., Van Schaftingen, J.: Boundary estimates for elliptic systems with $L^{1}$-data. Calc. Var. Partial Differential Equations 30, 369-388 (2007) Zbl 1149.35025 MR 2332419

[6] Cermelli, P., Leoni, G.: Energy and forces on dislocations. SIAM J. Math. Anal. 37, 11311160 (2005) Zbl 1141.74023 MR 2192291

[7] Conti, S., Ortiz, M.: Dislocation microstructures and the effective behavior of single crystals. Arch. Ration. Mech. Anal. 176, 103-147 (2005) Zbl 1064.74144 MR 2185859

[8] Dautray, R., Lions, J.-L.: Mathematical Analysis and Numerical Methods for Science and Technology. Vol. 3, Springer, Berlin (1988). Z Zbl 0944.47002 MR 1064315

[9] Deshpande, V. S., Needleman, A., Van der Giessen, E.: Finite strain discrete dislocation plasticity. In: Proceedings of a Symposium on Dynamic Failure and Thin Film Mechanics, J. Mech. Phys. Solids 51, 2057-2083 (2003) Zbl 1041.74504 MR 2048744

[10] Fleck, N. A., Hutchinson, J. W.: A phenomenological theory for strain gradient effects in plasticity. J. Mech. Phys. Solids 41, 1825-1857 (1993) Zbl 0791.73029 MR 1248268

[11] Garroni, A., Focardi, M.: A 1D phase field model for dislocations and a second order $\Gamma$-limit. SIAM Multiscale Model. Simul. 6, 1098-1124 (2007) Zbl 1152.82308 MR 2393027 
[12] Garroni, A., Müller, S.: A variational model for dislocations in the line tension limit. Arch. Ration. Mech. Anal. 181, 535-578 (2006) Zbl 1158.74365 MR 2231783

[13] Gurtin, M. E.: On the plasticity of single crystals: free energy, microforces, plastic-strain gradients. J. Mech. Phys. Solids 48, 989-1036 (2000) Zbl 0988.74021 MR 1746552

[14] Gurtin, M. E.: A gradient theory of single-crystal viscoplasticity that accounts for geometrically necessary dislocations. J. Mech. Phys. Solids 50, 5-32 (2002) Zbl 1043.74007 MR 1869436

[15] Gurtin, M. E., Anand, L.: A theory of strain-gradient plasticity for isotropic, plastically irrotational materials. I. Small deformations. J. Mech. Phys. Solids 53, 1624-1649 (2005) Zbl 1120.74353 MR 2146591

[16] Jerrard, R. L., Soner, H. M.: Limiting behavior of the Ginzburg-Landau functional. J. Funct. Anal. 192, 524-561 (2002) Zbl 1028.49015 MR 1923413

[17] Koslowski, M., Cuitiño, A. M., Ortiz, M.: A phase-field theory of dislocation dynamics, strain hardening and hysteresis in ductile single crystals. J. Mech. Phys. Solids 50, 2597-2635 (2002) Zbl 1094.74563 MR 1935021

[18] Nicola, L., Van der Giessen, E., Gurtin, M.: Effect of defect energy on strain-gradient predictions of confined single-crystal plasticity. J. Mech. Phys. Solids 53, 1280-1294 (2005) Zbl 1120.74358 MR 2137064

[19] Nye, J. F.: Some geometrical relations in dislocated crystals. Acta Metallurgica 1, 153-162 (1953)

[20] Ortiz, M., Repetto, E. A.: Nonconvex energy minimization and dislocation structures in ductile single crystals. J. Mech. Phys. Solids 47, 397-462 (1999) Zbl $0964.74012 \mid$ MR 1674064

[21] Ponsiglione, M.: Elastic energy stored in a crystal induced by screw dislocations: from discrete to continuous. SIAM J. Math. Anal. 39, 449-469 (2007) Zbl 1135.74037 MR 2338415

[22] Reshetnyak, Yu. G.: General theorems on semicontinuity and convergence with functionals. Sibirsk. Mat. Zh. 8, 1051-1069 (1967) (in Russian) Zbl 0179.20902 MR 0220127

[23] Sandier, E., Serfaty, S.: Limiting vorticities for the Ginzburg-Landau equations. Duke Math. J. 117, 403-446 (2003) Zbl 1035.82045 MR 1979050

[24] Sandier, E., Serfaty, S.: Vortices in the Magnetic Ginzburg-Landau Model. Progr. Nonlinear Differential Equations Appl. 70, Birkhäuser Boston, Boston, MA (2007) Zbl 1112.35002 MR 2279839

[25] Van Schaftingen, J.: Estimates for $L^{1}$-vector fields. C. R. Math. Acad. Sci. Paris 339, 181-186 (2004) Zbl 1049.35069 MR 2078071 